\title{
Knots in collapsible and non-collapsible balls
}

\author{
Bruno Benedetti* \\ Institut für Informatik \\ Freie Universität Berlin \\ Takustr. 9 \\ 14195 Berlin, Germany \\ bruno@zedat.fu-berlin.de
}

\author{
Frank H. Lutz ${ }^{\dagger}$ \\ Institut für Mathematik \\ Technische Universität Berlin \\ Straße des 17. Juni 136 \\ 10623 Berlin, Germany \\ lutz@math.tu-berlin.de
}

Submitted: Apr 24, 2013; Accepted: Aug 13, 2013; Published: Aug 30, 2013

Mathematics Subject Classifications: 57Q15, 52B22, 57M25

\begin{abstract}
We construct the first explicit example of a simplicial 3-ball $B_{15,66}$ that is not collapsible. It has only 15 vertices. We exhibit a second 3-ball $B_{12,38}$ with 12 vertices that is collapsible and not shellable, but evasive. Finally, we present the first explicit triangulation of a 3 -sphere $S_{18,125}$ (with only 18 vertices) that is not locally constructible. All these examples are based on knotted subcomplexes with only three edges; the knots are the trefoil, the double trefoil, and the triple trefoil, respectively. The more complicated the knot is, the more distant the triangulation is from being polytopal, collapsible, etc. Further consequences of our work are:

(1) Unshellable 3-spheres may have vertex-decomposable barycentric subdivisions. (This shows the strictness of an implication proven by Billera and Provan.)

(2) For $d$-balls, vertex-decomposable implies non-evasive implies collapsible, and for $d=3$ all implications are strict. (This answers a question by Barmak.)

(3) Locally constructible 3-balls may contain a double trefoil knot as a 3-edge subcomplex. (This improves a result of Benedetti and Ziegler.)

(4) Rudin's ball is non-evasive.
\end{abstract}

Keywords: knots in triangulations, shellability, local constructibility, non-evasiveness, collapsibility, discrete Morse theory

*Supported by the Swedish Research Council, grant "Triangulerade Mångfalder, Knutteori i diskrete Morseteori" and the DFG Collaborative Research Center TRR 109, "Discretization in Geometry and Dynamics".

†Supported by the DFG Research Group "Polyhedral Surfaces". 


\section{Introduction}

CollapsibiLity is a combinatorial property introduced by Whitehead, and somewhat stronger than contractibility. In 1964, Bing proved using knot theory that some triangulations of the 3-ball are not collapsible $[12,19]$. Bing's method works as follows. One starts with a finely-triangulated 3-ball embedded in the Euclidean 3-space. Then one drills a knot-shaped tubular hole inside it, stopping one step before destroying the property of being a 3-ball; see Figure 1. The resulting 3-ball contains a knot that consists of a single interior edge plus many boundary edges. This interior edge is usually called knotted spanning. If the knot is sufficiently complicated (like a double, or a triple trefoil), Bing's ball cannot be collapsible $[12,19]$; see also [8]. In contrast, if the knot is simple enough (like a single trefoil), then the Bing ball may be collapsible [25].

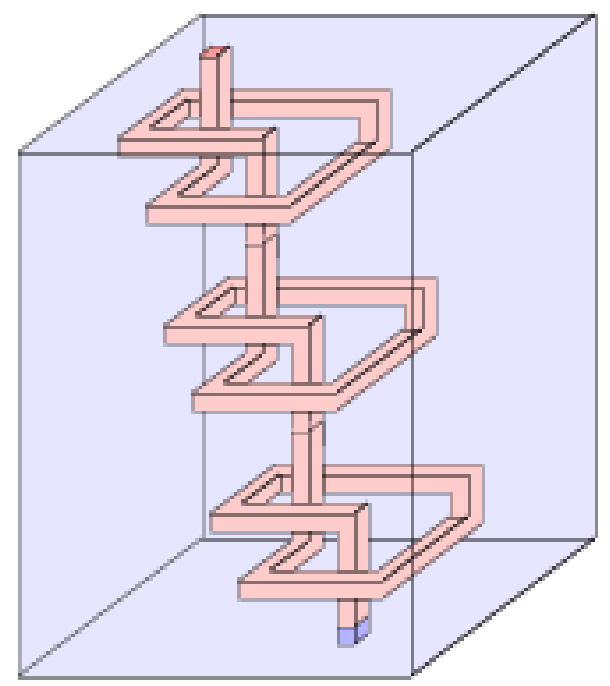

Figure 1: A triple trefoil drilled inside a ball, stopping one edge before perforating it, yields a non-collapsible 3-ball.

Thus the existence of a short knot in the triangulation prevents a 3-ball from having a desirable combinatorial property, namely, collapsibility. This turned out to be a recurrent motive in literature. In the Eighties, several authors asked whether all 3-spheres are shellable. This was answered in 1991 by Lickorish in the negative [24]: The presence in a 3-sphere of a triple trefoil on three edges prevents it from being shellable. It remained open whether all spheres are constructible (a slighly weaker property than shellability). However, in 2000 Hachimori and Ziegler [21] showed that the presence of any non-trivial knot on three vertices in a 3 -sphere even prevents it from being constructible. Finally, in 1994 the physicists Durhuus and Jonsson [15] asked whether all 3-spheres are locally constructible. Once again, a negative answer, based on Lickorish's original argument, was found using knot theory; see Benedetti-Ziegler [11]. 
These examples represent spheres that are far away from being polytopal. Thus, they are good candidates for testing properties that are true for polytopes, but only conjectured to be true for spheres. Moreover, they represent good test instances for algorithms in computational topology, as they are complicated triangulations of relatively simple spaces.

Unfortunately, the knotted counterexamples mentioned so far have a defect: They are easy to explain at the blackboard, but they yield triangulations with many vertices. The purpose of this paper is to come up with analogous 'test examples' that are smaller in size, but still contain topological obstructions that prevent them from having nice combinatorial properties.

A first idea to save on the number of faces is to start by realizing the respective knot in 3-space, using (curved) arcs. Obviously, any knot can be realized with exactly three $\operatorname{arcs}$ in $\mathbb{R}^{3}$ (we just need to draw it and insert three vertices along the knot). If we thicken the arcs into three 'bananas', the resulting 3-complex $P$ is homeomorphic to a solid torus pinched three times. By inserting 2-dimensional membranes, $P$ can be made contractible, and then it can be thickened to a 3-ball (or a 3-sphere) simply by adding cones. This approach costs a lot of manual effort, but a posteriori, it allows us to obtain new insight. In fact, here comes the second idea: We can ask a computer to perform random bistellar flips to the triangulation of the ball, without modifying the subcomplex $P$. Performing the flips according to a simulated annealing strategy [13] we were able to decrease the size of the triangulation, but for sure the flips will preserve the knotted substructure and its number of arcs.

This construction was introduced by the second author in [29], who applied it to the single trefoil, thereby obtaining a knotted 3-ball $B_{12,38}$ with 12 vertices and 38 tetrahedra. Here we apply the method to the double trefoil and the triple trefoil. The resulting spheres turn out to be interesting in connection with some properties which we will now describe.

The notion of EVASIVENESS has appeared first in theoretical computer science, in Karp's conjecture on monotone graph properties. Kahn, Saks and Sturtevant [23] extended the evasiveness property to simplicial complexes, showing that non-evasiveness strictly implies collapsibility. One can easily construct explicit examples of collapsible evasive 2-complexes in which none of the vertex-links is contractible [6]; see also [9]. Basically there are three known ways to prove that a certain complex $E$ is evasive:

(A) One shows that none of its vertex-links is contractible, cf. [6];

(B) one proves that the Alexander dual of $E$ is evasive, cf. [23];

(C) one shows (for example, via knot-theoretic arguments [12]) that $E$ is not even collapsible.

But are there collapsible evasive balls? And if so, how do we prove that they are evasive? Clearly, none of the approaches above would work. This was asked to us by Barmak (private communication). Once again, we found a counterexample in the realm of knotted triangulations: specifically, Lutz's triangulation $B_{12,38}$, which contains a single-trefoil knotted spanning edge.

Main Theorem 1. The 3-ball $B_{12,38}$ is collapsible and evasive. However, it is not shellable and not locally constructible. 
To prove collapsibility, we tried, using the computer, several collapsing sequences, until we found a lucky one. To show evasiveness, we used some sort of 'trick': We computed the homology of what would be left from $B_{12,38}$ after deleting roughly half of its vertices. It turns out that deleting five vertices from $B_{12,38}$ (no matter which ones) yields almost always some complex with non-trivial homology. From that we were able to exclude non-evasiveness.

En passant, we also prove the non-evasiveness of other existing triangulations that were known to be collapsible, like Rudin's ball (Theorem 6.3) or Lutz's triangulations $B_{7,10}[27]$ and $B_{9,18}[26]$.

Main Theorem 1 can be viewed as an improvement on the result from 1972 by Lickorish-Martin [25] and Hamstrom-Jerrard [22] that a ball with a knotted spanning edge can be collapsible. Recently Benedetti-Ziegler [11] constructed a similar example with all vertices on the boundary. In contrast, our $B_{12,38}$ has exactly one interior vertex. We also mention that $B_{12,38}$ is the first example of a manifold that admits a perfect discrete Morse function, but cannot admit a perfect Fourier-Morse function in the sense of Engström [17]. In fact, a complex is non-evasive if and only if it admits a Fourier-Morse function with only one critical cell.

VERTEX-DECOMPOSABILITY is a strengthening of shellability, much like non-evasiveness is a strengthening of collapsibility. It was introduced by Billera and Provan in 1980, in connection with the Hirsch conjecture [31]. For 3-balls, we have the following diagram of implications:

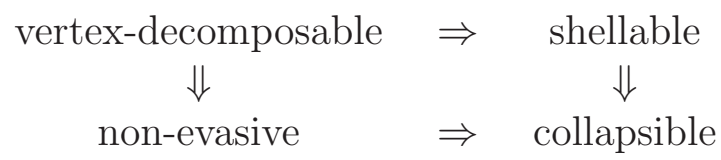

In addition, the barycentric subdivision of any shellable complex is vertex-decomposable [31] — and the barycentric subdivision of any collapsible complex is non-evasive [33]. What about the converse? Can an unshellable ball or sphere become vertex-decomposable after a single barycentric subdivision? The answer is positive. The barycentric subdivision of $B_{12,38}$ is, in fact, vertex-decomposable. The same holds for $S_{13,56}$, the unshellable 3sphere obtained coning off the boundary of $B_{12,38}$; see Proposition 6.8.

Next, we turn to a concrete question from Discrete QUANTUm GRAVITY. Suppose that we wish to take a walk on the various triangulations of $S^{3}$, by starting with the boundary of the 4-simplex and performing a random sequence of bistellar flips (also known as 'Pachner moves'). All triangulated 3 -spheres can be obtained this way [30], but some may be less likely to appear than others, like the 16-vertex triangulation $S_{16,104}$ by Dougherty, Faber and Murphy [14]; see also [5]. (In fact, any 'Pachner walk' from the boundary of the 4-simplex to $S_{16,104}$ must pass through spheres with more than 16 vertices.) This 'random Pachner walk' model is used in discrete quantum gravity, by Ambjørn, Durhuus, Jonsson and others, to estimate the total number of triangulations of $S^{3}[3,4]$. Durhuus and Jonsson have also developed the property of local constructibility, conjecturing it would hold for all 3-spheres [15]. As we said, the conjecture was negatively answered in [11], but it remained unclear how difficult it is to reach counterexamples, using a random Pachner walk. In other words: How outspread should the simulation be, before we have the chance 
to meet a non-locally constructible sphere?

Here we answer this question by presenting the first explicit triangulation of a nonlocally constructible 3 -sphere. For that, we have to adapt the construction of $B_{12,38}$ from the single trefoil to the triple trefoil. In the end, we manage to use only 18 vertices. The surprise is that via Pachner moves, the final triangulation is reachable rather straightforwardly.

Main Theorem 2. Some 17-vertex triangulation $B_{17,95}$ of the 3 -ball contains a triple trefoil knotted spanning edge. This $B_{17,95}$ is not collapsible. Coning off the boundary of $B_{17,95}$ one obtains a knotted 3 -sphere $S_{18,125}$ that is not locally constructible. Removing any tetrahedron from $S_{18,125}$ one obtains a knotted 3-ball that is neither locally constructible nor collapsible. This $S_{18,125}$ is '3-stellated', in the notation of Bagchi-Datta [5]: it can be reduced to the boundary of a 4-simplex by using 94 Pachner moves that do not add further vertices.

After dealing with the single trefoil and the triple trefoil, let us turn to the intermediate case of the double trefoil. By the work of Benedetti-Ziegler, any 3-ball containing a 3-edge knot in its 1-skeleton cannot be locally constructible if the knot is the sum of three or more trefoils [11]. But is this bound best possible? In [11] it is shown with topological arguments that a collapsible 3-ball may contain a double trefoil knot on 3 edges. Recall that locally constructible 3-balls are characterized by the property of collapsing onto their boundary minus a triangle [11]. This is stronger than just being collapsible. It remained unclear whether a locally constructible 3-ball may indeed contain a double trefoil on three edges.

We answer this question affirmatively in Section 4. As before, the key consists in triangulating cleverly, so that computational approaches may succeed. On the way to this result, we produce a smaller example of a non-collapsible ball, using only 15 vertices and 66 tetrahedra.

Main Theorem 3. Some 15-vertex triangulation $B_{15,66}$ of the 3-ball contains a double trefoil knotted spanning edge. This $B_{15,66}$ is not collapsible. Coning off the boundary of $B_{15,66}$ one obtains a knotted 3 -sphere $S_{16,92}$ that is locally constructible. Removing the tetrahedron 191415 from $S_{16,92}$ one obtains a knotted 3-ball that is collapsible and locally constructible.

Now, for each $d \geqslant 3$ one has the following hierarchy of combinatorial properties of triangulated $d$-spheres [11]:

$\{$ vertex-decomposable $\} \subsetneq\{$ shellable $\} \subseteq\{$ constructible $\} \subsetneq\{$ LC $\} \subsetneq\{$ all $d$-spheres $\}$.

An analogous hierarchy holds for $d$-balls $(d \geqslant 3)[11]$ :

$$
\begin{aligned}
\text { \{vertex-decomposable }\} & \subsetneq\{\text { shellable }\} \subsetneq\{\text { constructible }\} \subsetneq\{\mathrm{LC}\} \\
& \subsetneq\left\{\begin{array}{l}
\text { collapsible onto } \\
(d-2) \text {-complex }
\end{array}\right\} \subsetneq\{\text { all } d \text {-balls }\} .
\end{aligned}
$$


Table 1: List of 3-balls and 3-spheres and their properties.

\begin{tabular}{c|llllll}
\hline Trefoils & \multicolumn{3}{c|}{ 3-ball $B$} & \multicolumn{2}{c}{ 3-Sphere $\partial(v * B)$} & \multicolumn{2}{c}{ 3-ball $\partial(v * B)-\Sigma$} \\
\hline 0 & $B_{7,10}$ & sh., NE, non-VD & $S_{8,20}$ & VD & $B_{8,19}$ & VD \\
0 & $B_{8,13}$ & sh., non-VD & $S_{9,25}$ & sh., non-VD & $B_{9,24}$ & sh. \\
0 & $B_{9,18}$ & constr., NE, non-sh. & $S_{10,32}$ & sh. & $B_{10,31}$ & sh. \\
1 & $B_{12,38}$ & coll., evasive, non-LC & $S_{13,56}$ & LC, non-constr. & $B_{13,55}$ & LC, non-constr. \\
2 & $B_{15,66}$ & non-coll. & $S_{16,92}$ & LC, non-constr. & $B_{16,91}$ & LC, non-constr. \\
3 & $B_{17,95}$ & non-coll. & $S_{18,125}$ & non-LC & $B_{18,124}$ & non-coll.
\end{tabular}

Note: $\mathrm{VD}=$ vertex-decomposable, sh. = shellable, constr. $=$ constructible, $\mathrm{LC}=$ locally constructible, coll. $=$ collapsible, $\mathrm{NE}=$ non-evasive. "Trefolls: $t$ " means "containing a $t$-fold trefoil on 3 edges".

(When $d=3$, "collapsible onto a 1-complex" is equivalent to "collapsible".)

Here is another interesting hierarchy for balls, which can be merged with the previous one.

Main Theorem 4. There are the following inclusion relations between families of simplicial d-balls:

$$
\{\text { vertex-decomposable }\} \subseteq\{\text { non-evasive }\} \subseteq\{\text { collapsible }\} \subseteq\{\text { all d-balls }\} .
$$

For 2-balls all inclusions above are equalities, whereas for 3-balls all inclusions above are strict. More precisely, we have the following 'mixed' hierarchy:

$$
\begin{aligned}
\text { \{vertex-decomposable }\} & \subsetneq\left\{\begin{array}{c}
\text { shellable } A N D \\
\text { non-evasive }
\end{array}\right\} \subsetneq\left\{\begin{array}{c}
\text { shellable } O R \\
\text { non-evasive }
\end{array}\right\} \\
& \subsetneq\{\text { collapsible }\} \subsetneq\{\text { all } 3 \text {-balls }\} .
\end{aligned}
$$

\section{Background}

\subsection{Combinatorial properties of triangulated spheres and balls}

A $d$-complex is pure if all of its top-dimensional faces (called facets) have the same dimension.

A pure $d$-complex $C$ is constructible if either (1) $C$ is a simplex, or (2) $C$ is a disjoint union of points, or (3) $d \geqslant 1$ and $C$ can be written as $C=C_{1} \cup C_{2}$, where $C_{1}$ and $C_{2}$ are constructible $d$-complexes and $C_{1} \cap C_{2}$ is a constructible $(d-1)$-complex.

A pure $d$-complex $C$ is shellable if either (1) $C$ is a simplex, or (2) $C$ is a disjoint union of points, or (3) $d \geqslant 1$ and $C$ can be written as $C=C_{1} \cup C_{2}$, where $C_{1}$ is a shellable $d$-complex, $C_{2}$ is a $d$-simplex, and $C_{1} \cap C_{2}$ is a shellable $(d-1)$-complex.

A pure $d$-complex $C$ is vertex-decomposable if either (1) $C$ is a simplex, or (2) $C$ is a disjoint union of points, or (3) $d \geqslant 1$ and there is a vertex $v$ in $C$ (called shedding vertex) 
such that $\operatorname{del}(v, C)$ and $\operatorname{link}(v, C)$ are both vertex-decomposable (and $\operatorname{del}(v, C)$ is pure $d$-dimensional).

A (not necessarily pure!) $d$-complex $C$ is non-evasive if either $(1) C$ is a simplex, or (2) $C$ is a single point, or $(3) d \geqslant 1$ and there is a vertex $v$ in $C$ such that $\operatorname{del}(v, C)$ and $\operatorname{link}(v, C)$ are both non-evasive.

An elementary collapse is the simultaneous removal from a $d$-complex $C$ of a pair of faces $(\sigma, \Sigma)$ with the prerogative that $\Sigma$ is the only face properly containing $\sigma$. (This condition is usually abbreviated in the expression ' $\sigma$ is a free face of $\Sigma$ '; some complexes have no free face). If $C^{\prime}:=C-\Sigma-\sigma$, we say that the complex $C$ collapses onto the complex $C^{\prime}$. Even if $C$ is pure, this $C^{\prime}$ need not be pure. We say that the complex $C$ collapses onto $D$ if $C$ can be reduced to $D$ by some finite sequence of elementary collapses. A (not necessarily pure) $d$-complex $C$ is collapsible if it collapses onto a single vertex.

A simplicial 3-ball is locally constructible (or shortly $L C$ ) if it can be collapsed onto its boundary minus a triangle. A simplicial 3-sphere is locally constructible (or shortly $L C$ ) if the removal of some tetrahedron makes it collapsible onto one of its vertices.

\subsection{Perfect discrete Morse functions}

A map $f: C \rightarrow \mathbb{R}$ on a simplicial complex $C$ is a discrete Morse function on $C$ if for each face $\sigma$

(i) there is at most one boundary facet $\rho$ of $\sigma$ such that $f(\rho) \geqslant f(\sigma)$ and

(ii) there is at most one face $\tau$ having $\sigma$ as boundary facet such that $f(\tau) \leqslant f(\sigma)$.

A critical face of $f$ is a face of $C$ for which

(i) there is no boundary facet $\rho$ of $\sigma$ such that $f(\rho) \geqslant f(\sigma)$ and

(ii) there is no face $\tau$ having $\sigma$ as boundary facet such that $f(\tau) \leqslant f(\sigma)$.

A collapse-pair of $f$ is a pair of faces $(\sigma, \tau)$ such that

(i) $\sigma$ is a boundary facet of $\tau$ and

(ii) $f(\sigma) \geqslant f(\tau)$.

Forman [18, Section 2] showed that for each discrete Morse function $f$ the collapse pairs of $f$ form a partial matching of the face poset of $C$. The unmatched faces are precisely the critical faces of $f$. Each complex $C$ endowed with a discrete Morse function is homotopy equivalent to a cell complex with exactly one cell of dimension $i$ for each critical $i$-face [18]. In particular, if we denote by $c_{i}(f)$ the number of critical $i$-faces of $f$, and by $\beta_{i}(C)$ the $i$-th Betti number of $C$, one has

$$
c_{i}(f) \geqslant \beta_{i}(C)
$$

for all discrete Morse functions $f$ on $C$. These inequalities need not be sharp. If they are sharp for all $i$, the discrete Morse function is called perfect. However, for each $k$ and for each $d \geqslant 3$ there is a $d$-sphere $S[8]$ such that for any discrete Morse function $f$ on $S$, one has

$$
c_{d-1}(f) \geqslant k+\beta_{d-1}(S)=k .
$$




\subsection{Knots and knot-theoretic obstructions}

A knot is a simple closed curve in a 3-sphere. All the knots we consider are tame, that is, realizable as 1-dimensional subcomplexes of some triangulated 3-sphere. A knot is trivial if it bounds a disc; all the knots we consider here are non-trivial. The knot group is the fundamental group of the knot complement inside the ambient sphere. For example, the knot group of the trefoil knot (and of its mirror image) is $\left\langle x, y \mid x^{2}=y^{3}\right\rangle$. Ambient isotopic knots have isomorphic knot groups. A connected sum of two knots is a knot obtained by cutting out a tiny arc from each and then sewing the resulting curves together along the boundary of the cutouts. For example, summing two trefoils one obtains the "granny knot"; summing a trefoil and its mirror image one obtains the so-called "square knot". When we say "double trefoil", we mean any of these (granny knot or square knot): From the point of view of the knot group, it does not matter. A knot is m-complicated if the knot group has a presentation with $m+1$ generators, but no presentation with $m$ generators. By "at least $m$-complicated" we mean " $k$-complicated for some $k \geqslant m$ ". There exist arbitrarily complicated knots: Goodrick [19] showed that the connected sum of $m$ trefoil knots is at least $m$-complicated.

A spanning edge of a 3-ball $B$ is an interior edge that has both endpoints on the boundary $\partial B$. An $\mathfrak{L}$-knotted spanning edge of a 3 -ball $B$ is a spanning edge $x y$ such that some simple path on $\partial B$ between $x$ and $y$ completes the edge to a (non-trivial) knot $\mathfrak{L}$. From the simply-connectedness of 2-spheres it follows that the knot type does not depend on the boundary path chosen; in other words, the knot is determined by the edge. More generally, a spanning arc is a path of interior edges in a 3 -ball $B$, such that both extremes of the path lie on the boundary $\partial B$. If every path on $\partial B$ between the two endpoints of a spanning arc completes the latter to a knot $\mathfrak{L}$, the arc is called $\mathfrak{L}$-knotted. Note that the relative interior of the arc is allowed to intersect the boundary of the 3-ball; compare Ehrenborg-Hachimori [16].

Below is a list of known results on knotted spheres and balls. As for the notation, if $B$ is a 3 -ball with a knotted spanning edge, by $S_{B}$ we will mean the 3 -sphere $\partial(v * B)$, where $v$ is a new vertex. By $\mathfrak{L}_{t}$ we denote a connected sum of $t$ trefoil knots.

Theorem 2.1 (Benedetti/Ehrenborg/Hachimori/Ziegler). Any 3-ball with an $\mathfrak{L}_{t}$-knotted spanning arc of $t$ edges cannot be $L C$ [8], but it can be collapsible [11, 25]. An arbitrary 3 -ball with an $\mathfrak{L}_{1}$-knotted spanning arc of less than 3 edges cannot be shellable nor constructible [21]. In contrast, some shellable 3 -balls have a $\mathfrak{L}_{1}$-knotted spanning arc of 3 edges [21].

Theorem 2.2 (Adams et al. [1, Theorem 7.1]). Any knotted 3-ball in which the knot $\mathfrak{L}_{t}$ is realized with e edges cannot be rectilinearly embeddable in $\mathbb{R}^{3}$ if $e \leqslant 2 t+3$.

Theorem 2.3 (Benedetti/Ehrenborg/Hachimori/Shimokawa/Ziegler). A 3-sphere or a 3-ball, with a subcomplex of $m$ edges, isotopic to the sum of $t$ trefoil knots,

- cannot be vertex-decomposable if $t \geqslant\left\lfloor\frac{m}{3}\right\rfloor$ [21],

- cannot be constructible/shellable if $t \geqslant\left\lfloor\frac{m}{2}\right\rfloor[16,20]$, and

- cannot be $L C$ if $t \geqslant m[11]$. 
The first two bounds are known to be sharp [16, 21]; the latter bound is also sharp, as far as spheres are concerned [7, 11].

Theorem 2.4 (Benedetti/Lickorish [8, 24]). Let $S$ be a 3-sphere with a subcomplex of $m$ edges, isotopic to the sum of trefoil knots. For any discrete Morse function $f$ on $S$, one has

$$
c_{2}(f) \geqslant t-m+1
$$

\section{The single trefoil}

In this section, we study the 3-ball $B_{12,38}$ introduced in [29] and given by the following 38 facets:

$\begin{array}{llllllll}2347, & 23410, & 23710, & 2457, & 24510, & 25713, & 25810, & 25813, \\ 26911, & 261113, & 261213, & 27810, & 27811, & 271113, & 28911, & 28912, \\ 281213, & 3467, & 34610, & 35813, & 35911, & 35913, & 36712, & 361013, \\ 361213, & 371012, & 38911, & 38912, & 381213, & 391012, & 391013, & 4567, \\ 45610, & 5679, & 56911, & 561011, & 57913, & 6101113 . & & \end{array}$

The ball is contructed in a way such that the edge 23 is a knotted spanning edge for $B_{12,38}$, the knot being a single trefoil. In particular, by Theorem 2.1, $B_{12,38}$ is not shellable, not constructible and not LC. Here we show that:

(1) $B_{12,38}$ is not rectilinearly-embeddable in $\mathbb{R}^{3}$;

(2) $B_{12,38}$ is evasive;

(3) $B_{12,38}$ is collapsible;

(4) The 3-sphere $\partial\left(1 * B_{12,38}\right)$ minus the facet 1269 is an LC knotted 3-ball.

Proposition 3.1. $B_{12,38}$ is not rectilinearly-embeddable in $\mathbb{R}^{3}$.

Proof. The boundary of $B_{12,38}$ consists of the following 18 triangles:

$$
\begin{aligned}
& 269, \quad 2612, \quad 2912, \quad 358, \quad 3511, \quad 3811, \quad 5810, \quad 51011, \quad 679 \text {, } \\
& 6712,7810,7811,7913,71012,71113,91012,91013,101113 .
\end{aligned}
$$

In particular, the four edges $26,67,78$ and 38 form a boundary path from the vertex 2 to the vertex 3 . Together with the interior edge 23 , this path closes up to a pentagonal trefoil knot. By Theorem 2.2, $B_{12,38}$ cannot be rectilinearly embedded in $\mathbb{R}^{3}$, because the stick number of the trefoil knot is 6 .

Proposition 3.2. $B_{12,38}$ is collapsible, but not $L C$.

Proof. By Theorem 2.1, $B$ is not LC; in particular, $B$ does not collapse onto its boundary minus a triangle. So, in the first phase of the collapse (the one in which the tetrahedra are collapsed away) we have to remove several boundary triangles in order to succeed. Now, finding a collapse can be difficult, but verifying the correctness of a given collapse is fast. The following is a certificate of the collapsibility of $B_{12,38}$. 
First phase (pairs "triangle" $\rightarrow$ "tetrahedron"):

\begin{tabular}{|c|c|c|c|c|c|c|c|c|c|}
\hline 101113 & $\rightarrow 6101113$ & 7913 & $\rightarrow 57913$ & 61011 & $\rightarrow$ & 561011 & 5611 & $\rightarrow$ & 56911 \\
\hline 2612 & $\rightarrow 261213$ & 579 & $\rightarrow 5679$ & 91012 & $\rightarrow$ & 391012 , & 71113 & $\rightarrow$ & 271113 , \\
\hline 5911 & $\rightarrow 35911$ & 2713 & 25713 , & 3912 & $\rightarrow$ & 38912 , & 2613 & $\rightarrow$ & 261113 , \\
\hline 3812 & $\rightarrow 381213$ & 3911 & $\rightarrow 38911$ & 71012 & $\rightarrow$ & 371012 , & 8912 & $\rightarrow$ & 28912 \\
\hline 61013 & $\rightarrow 361013$ & 358 & $\rightarrow 35813$ & 6911 & $\rightarrow$ & 26911 & 81213 & $\rightarrow$ & 281213 , \\
\hline 3613 & $\rightarrow 361213$ & 31013 & $\rightarrow 391013$ & 3513 & $\rightarrow$ & 35913 & 6712 & $\rightarrow$ & 36712 , \\
\hline 367 & $\rightarrow 3467$ & 567 & $\rightarrow 4567$ & 7811 & $\rightarrow$ & 27811 & 2911 & $\rightarrow$ & 28911 \\
\hline 346 & $\rightarrow 34610$ & 457 & $\rightarrow 2457$ & 5610 & $\rightarrow$ & 45610 & 3410 & $\rightarrow$ & 23410 \\
\hline 247 & $\rightarrow 2347$ & 237 & $\rightarrow 23710$ & 5810 & $\rightarrow$ & 25810 & 5813 & $\rightarrow$ & 25813 \\
\hline 7810 & $\rightarrow 27810$ & 245 & $\rightarrow 24510$ & & & & & & \\
\hline
\end{tabular}

Second phase (pairs "edge" $\rightarrow$ "triangle"):

\begin{tabular}{|c|c|c|c|c|c|c|c|c|c|c|c|c|}
\hline 812 & $\rightarrow 2812$ & 78 & $\rightarrow$ & 278 & 713 & $\rightarrow$ & 5713 & 810 & $\rightarrow$ & 2810 & 911 & $\rightarrow 8911$ \\
\hline 79 & $\rightarrow 679$ & 1011 & $\rightarrow$ & 51011 & 711 & $\rightarrow$ & 2711 & 58 & $\rightarrow$ & 258 & 912 & $\rightarrow 2912$ \\
\hline 712 & $\rightarrow 3712$ & 511 & $\rightarrow$ & 3511 & 35 & $\rightarrow$ & 359 & 57 & $\rightarrow$ & 257 & 1012 & $\rightarrow 31012$, \\
\hline 311 & $\rightarrow 3811$ & 67 & $\rightarrow$ & 467 & 47 & $\rightarrow$ & 347 & 27 & $\rightarrow$ & 2710 & 811 & $\rightarrow 2811$ \\
\hline 212 & $\rightarrow 21213$ & 1013 & $\rightarrow$ & 91013 , & 34 & $\rightarrow$ & 234 & 23 & $\rightarrow$ & 2310 & 710 & $\rightarrow 3710$ \\
\hline 910 & $\rightarrow 3910$ & 310 & $\rightarrow$ & 3610 & 610 & $\rightarrow$ & 4610 & 46 & $\rightarrow$ & 456 & 45 & $\rightarrow 4510$ \\
\hline 24 & $\rightarrow 2410$ & 36 & $\rightarrow$ & 3612 , & 210 & $\rightarrow$ & 2510 & 312 & $\rightarrow$ & 31213 , & 1213 & $\rightarrow 61213$ \\
\hline 25 & $\rightarrow \quad 2513$ & 56 & $\rightarrow$ & 569 & 613 & $\rightarrow$ & 61113 & 513 & $\rightarrow$ & 5913 & 1113 & $\rightarrow 21113$ \\
\hline 213 & $\rightarrow 2813$ & 913 & $\rightarrow$ & 3913, & 69 & $\rightarrow$ & 269 & 39 & $\rightarrow$ & 389 & 38 & $\rightarrow 3813$ \\
\hline
\end{tabular}

Third phase (pairs "vertex" $\rightarrow$ "edge"):

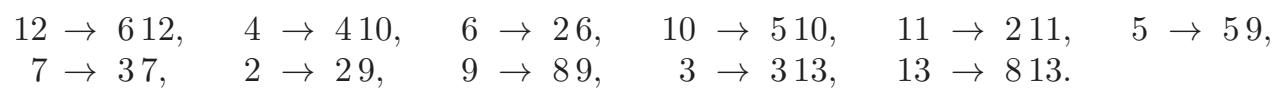

The above collapsing sequence was found with the randomized approach of [10].

Proposition 3.3. $B_{12,38}$ is evasive.

Proof. Let us establish some notation first. We identify each vertex of $B_{12,38}$ with its label, which is an integer in $A:=\{2, \ldots, 13\}$. For each subset $S$ of $A$, we denote by $C_{S}$ the complex obtained from $B_{12,38}$ by deleting the vertices in $S$.

Now, suppose by contradiction that $B$ is non-evasive. The vertices of $B_{12,38}$ can be reordered so that their progressive deletions and links are non-evasive. In particular, there exists a five-element subset $F$ of $A$ such that $C_{F}$ is non-evasive.

With the help of a computer program, we checked the homologies of all complexes obtained by deleting five vertices from $B$. Since the order of deletion does not matter, there are only $\left(\begin{array}{c}12 \\ 5\end{array}\right)=792$ cases to check, so the computation is extremely fast. It turns out that these homologies are never trivial, except for the following three cases:

(1) $F_{1}=\{4,5,8,10,11\}$

(2) $F_{2}=\{4,5,10,11,12\}$,

(3) $F_{3}=\{4,6,7,9,12\}$. 


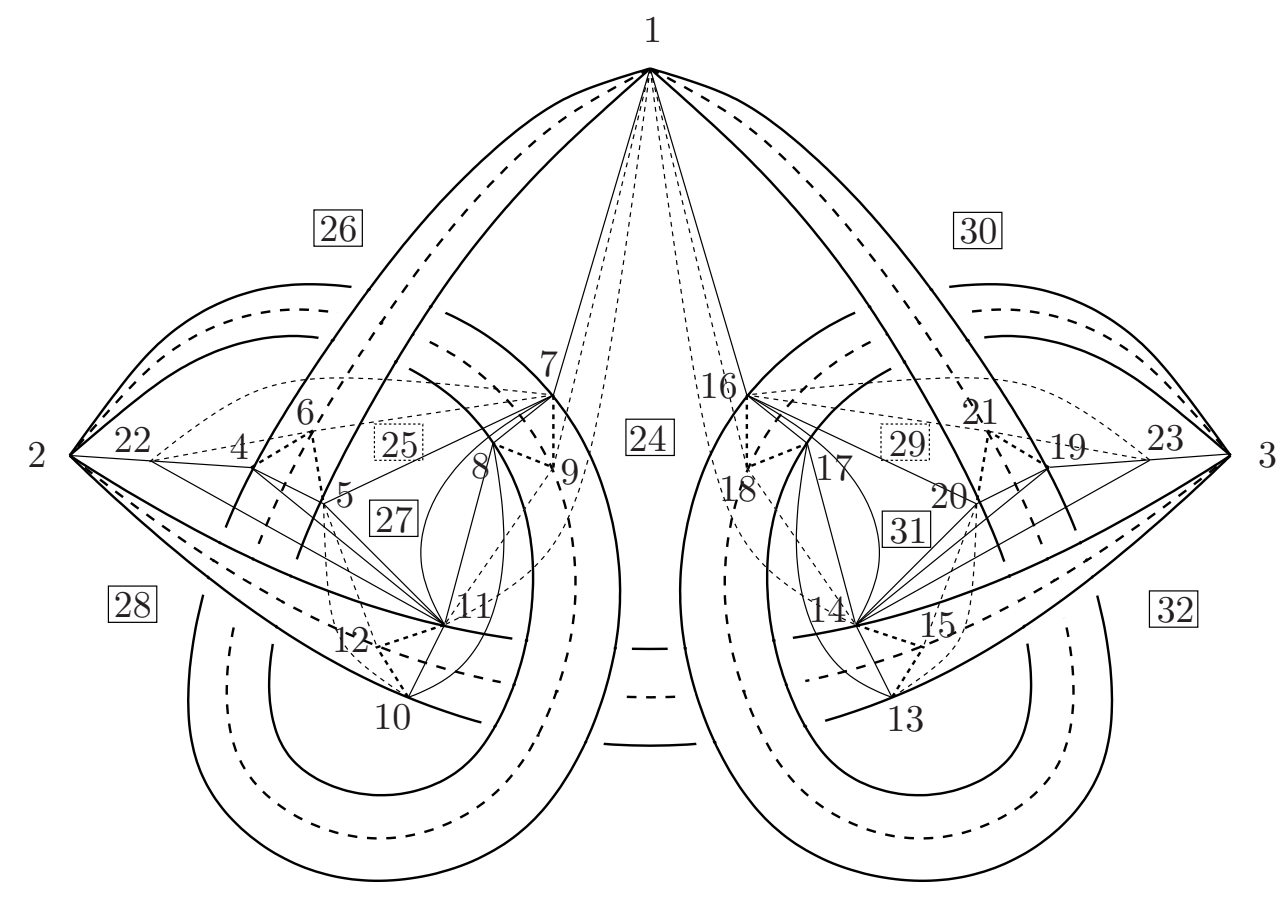

Figure 2: The double trefoil in the sphere $S_{33,192}$.

So, the non-evasive complex $C_{F}$ whose existence was postulated above must be either $C_{F_{1}}$, or $C_{F_{2}}$, or $C_{F_{3}}$. However, it is easy to see that the deletion of any vertex from $C_{F_{1}}$ yields a non-acyclic complex. The same holds for $C_{F_{2}}$ and $C_{F_{3}}$. Therefore, all three complexes $C_{F_{1}}, C_{F_{2}}$ and $C_{F_{3}}$ are evasive: A contradiction.

Remark 3.4. Let $S_{B}$ be the sphere obtained by coning off the boundary of $B_{12,38}$ with an extra vertex, labeled by 1 . Let $\Sigma$ be the tetrahedron 1269 and let $\sigma$ be its facet 269 . With the help of the computer, one can check that $S_{B}-\Sigma$ collapses onto the 2-ball $D$ consisting of the triangles 126,129 and 169. Since $D=\partial \Sigma-\sigma=\partial\left(S_{B}-\Sigma\right)-\sigma$, it follows that the knotted 3-ball $S_{B}-\Sigma$ is locally constructible (because it collapses onto its boundary minus the triangle $\sigma$ ). For a proof, see [7].

\section{The double trefoil}

In the following, we present the construction of a triangulated 3-sphere that contains a double trefoil knot on three edges in its 1-skeleton. In fact, there are two different ways to form the connected sum of two trefoil knots, the granny and the square knot. We base our construction on the square knot.

Let $12,23,13$ be the three edges forming the square knot, which, for our purposes, we simply call the double trefoil knot. An embedding of the knot in $\mathbb{R}^{3}$ is depicted in Figure 2. 

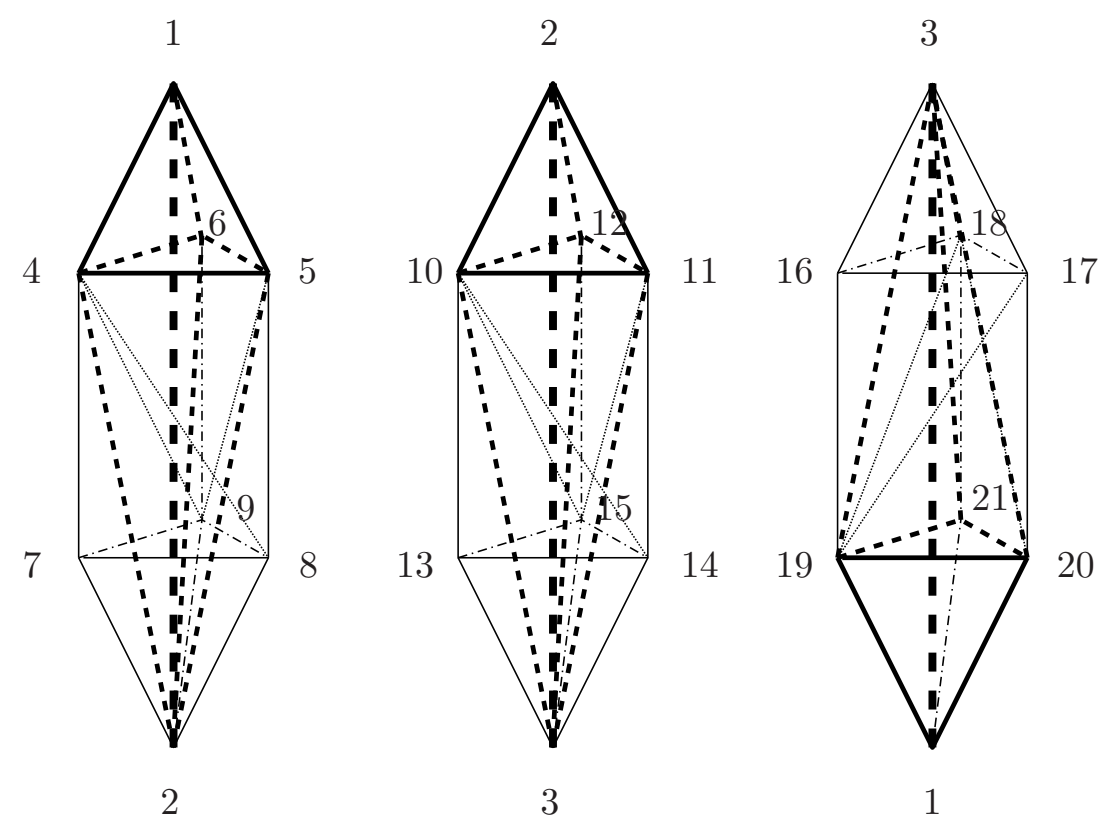

Figure 3: The spindles of $S_{33,192}$.

Our strategy is to place the knot into the 1-skeleton of a triangulated 3-dimensional sphere as follows.

- We start with an embedding of the knot in $\mathbb{R}^{3}$,

- we triangulate the region around the knot to obtain a triangulated 3-ball,

- we complete it to a triangulation of $S^{3}$ by adding the cone over its boundary.

Once the knot edges $12,23,13$ are placed in $\mathbb{R}^{3}$ we need to shield off these edges to prevent unwanted identifications of distant vertices later on. We protect each of the knot edges by placing a spindle around it; see Figure 3 for images of the spindles and Table 2 for lists of nine tetrahedra each, which form the three spindles. The additional vertices on the boundaries of the spindles allow us to close the holes of the knot by gluing in (triangulated) membrane patches.

Table 2: Part I of the sphere $S_{33,192}$ : The three spindles.

\begin{tabular}{|c|c|c|c|c|c|}
\hline 1245 & 2478 & 231011 & 3101314 & 131920 & 3161719 \\
\hline 1246 & 2458 & 231012 & 3101114 & 131921 & 3171920 \\
\hline \multirow[t]{4}{*}{1256} & 2589 & 231112 & 3111415 & 132021 & 3171820 \\
\hline & 2569 & & 3111215 & & 3182021 \\
\hline & 2479 & & 3101315 & & 3161819 \\
\hline & 2469 & & 3101215 & & 3181921 \\
\hline
\end{tabular}


Table 3: The triangles of the membranes in the sphere $S_{33,192}$.

\begin{tabular}{llllll}
\hline & & & & & \\
4511 & 157 & 1911 & 114 & 11620 & 141920 \\
41122 & 5711 & 179 & 11618 & 141620 & 141923 \\
21122 & 7811 & 8911 & 141718 & 141617 & 31423 \\
2722 & 81011 & & & 131417 & 31623 \\
6722 & 5810 & & & 131720 & 162123 \\
4622 & 51012 & & & 131520 & 192123 \\
567 & 51112 & & & 141520 & 162021 \\
\hline
\end{tabular}

In Figure 2, the diagonal edges on the boundaries of the spindles and also the interior edges of the spindles are not shown. All that we need at the moment are the vertices on the boundaries of the spindles. For example, if we move along the left spindle 1-2 from apex 1 to apex 2, we first meet the vertices $4,5,6$ and then the vertices $7,8,9$ on the spindle boundary.

The membrane patches can be read off from Table 3. The central triangle 11114 connects the left part with the right part of Figure 2 and contributes to the closure of the upper central hole. Next to the triangle 11114 on the left hand side in Figure 2 is the triangle 1911 from the third column of Table 3, followed by triangle 179 and so on. Once all the membrane triangles of Table 3 are in place in Figure 2, the resulting complex is a mixed 2- and 3-dimensional simplicial complex, consisting of spindle tetrahedra and membrane triangles. Since we closed all holes of the initial double trefoil knot, the resulting complex is contractible.

Our next aim is to thicken the intermediate mixed 2- and 3-dimensional complex to a triangulated 3-ball $B_{32,140}$. For this end we add local cones to Figure 2 with respect to the nine new vertices $24,25, \ldots, 32$. These cones are listed in Table 4 , the positions of their apices are marked in Figure 2 by boxes containing the new vertices.

If we add together all the (spindle) tetrahedra from Table 2 (Part I of the sphere $S_{33,192}$ ) with all the (cone) tetrahedra from Table 4 (Part II of the sphere $S_{33,192}$ ), we obtain a triangulated 3-ball $B_{32,140}$ with 32 vertices and 140 tetrahedral facets. By construction, the 3-ball $B_{32,140}$ contains the double trefoil knot in its 1-skeleton and all the membrane triangles in its 2-skeleton.

In a final step, we add to the 3 -ball $B_{32,140}$ the cone over its boundary with respect the vertex 33 (Part III of the sphere $S_{33,192}$ with tetrahedra as listed in Table 5) to obtain the 3 -sphere $S_{33,192}$. 
Table 4: Part II of the sphere $S_{33,192}$ : Tetrahedra to be added to Part I to obtain a ball $B_{32,140}$.

\begin{tabular}{lllllll}
\hline 462425 & 17926 & 152627 & 17924 & 19212429 & 1161830 & 1203031 \\
562425 & 27926 & 5112627 & 191124 & 20212429 & 3161830 & 14203031 \\
5102425 & 1567 & 10112627 & 891124 & 13202429 & 1162021 & 13143031 \\
5101225 & 16726 & 8101127 & 8101124 & 13152029 & 1162130 & 13141731 \\
5111225 & 672226 & 781127 & 581024 & 14152029 & 16212330 & 14161731 \\
571125 & 272226 & 571127 & 56924 & 14162029 & 3162330 & 14162031 \\
781125 & 14626 & 15727 & 58924 & 14161729 & 1192130 & 1162031 \\
891125 & 462226 & & 46924 & 14171829 & 19212330 & \\
56725 & 14526 & 8102728 & 47924 & 16202129 & 1192030 & 13173132 \\
672225 & 451126 & 581028 & & 16212329 & 14192030 & 13172032 \\
462225 & 4112226 & 5101228 & 1111424 & 19212329 & 14192330 & 13152032 \\
272225 & 2112226 & 5111228 & 10111424 & 3162329 & 3142330 & 14152032 \\
27825 & 2101126 & 451128 & 10131424 & 3161729 & 3131430 & 14192032 \\
28925 & & 4112228 & & 3171829 & & 14192332 \\
& & 2112228 & 1161824 & & & 3142332 \\
& 2111228 & 1141824 & & & 3131532 \\
& 2101228 & 14171824 & & & 3133032 \\
& & 2102628 & 13141724 & & & 13303132 \\
& & 10262728 & 13172024 & & & \\
\hline
\end{tabular}

Proposition 4.1. The 3-sphere $S_{33,192}$ consists of 192 tetrahedra and 33 vertices. It has face vector $f=(33,225,384,192)$ and contains the double trefoil knot on three edges in its 1-skeleton.

The 3-sphere $S_{33,192}$ is not minimal with the property of containing the double trefoil knot in its 1-skeleton. One way of obtaining smaller triangulations is by applying bistellar flips, cf. [13], to the triangulation $S_{33,192}$. If we want to keep the knot while doing local bistellar modifications on the triangulation, we merely have to exclude the knot edges 12, 23, 13 as pivot edges in the bistellar flip program BISTELLAR [28]. The smallest triangulation we found this way is $S_{16,92}$; see Table 6 for the list of facets of $S_{16,92}$.

Theorem 4.2. The 3 -sphere $S_{16,92}$ has 92 tetrahedra and 16 vertices. It has face vector $f=(16,108,184,92)$ and contains the double trefoil knot on three edges in its 1-skeleton. 
Table 5: Part III of the sphere $S_{33,192}$ : Cone over the boundary of the ball $B_{32,140}$.

\begin{tabular}{lllllll}
\hline 172433 & 172733 & 191133 & 192633 & 1111433 & 1141833 & 1162433 \\
1163133 & 1183033 & 1262733 & 1303133 & 292533 & 292633 & 2222533 \\
2222833 & 2262833 & 3182933 & 3183033 & 3232933 & 3233233 & 3303233 \\
47833 & 472433 & 482833 & 4222533 & 4222833 & 4242533 & 782733 \\
8272833 & 9112533 & 10121533 & 10122533 & 10131533 & 10132433 & 10242533 \\
11121533 & 11122533 & 11141533 & 13152933 & 13242933 & 14152933 & 14182933 \\
16171933 & 16173133 & 16192433 & 17193233 & 17313233 & 19232933 & 19233233 \\
19242933 & 26272833 & 30313233 & & & & \\
\hline
\end{tabular}

Table 6: The sphere $S_{16,92}$.

\begin{tabular}{llllllll}
\hline 1256 & 12512 & 12612 & 1378 & 13711 & 13811 & 1456 & 14516 \\
14612 & 141013 & 141016 & 141213 & 151213 & 151316 & 1789 & 17911 \\
18914 & 181014 & 181015 & 181115 & 191115 & 191415 & 1101314 & 1101516 \\
1131416 & 1141516 & 23413 & 23415 & 231315 & 2478 & 24715 & 24816 \\
241013 & 241016 & 25614 & 251214 & 26812 & 26816 & 26914 & 26916 \\
2789 & 27910 & 271013 & 271315 & 28914 & 281214 & 291016 & 341213 \\
341215 & 3567 & 35614 & 3578 & 35811 & 351114 & 36716 & 36914 \\
36916 & 371114 & 371416 & 391213 & 391216 & 391315 & 391415 & 3121516 \\
3141516 & 4567 & 4578 & 45816 & 46715 & 461215 & 581113 & 581316 \\
5111213 & 5111214 & 671315 & 671316 & 681215 & 681315 & 681316 & 791012 \\
791112 & 7101214 & 7101314 & 7111214 & 7131416 & 8101214 & 8101215 & 8111315 \\
9101216 & 9111213 & 9111315 & 10121516 & & & & \\
\hline
\end{tabular}

If we remove from the 3 -sphere $S_{16,92}$ the facet 191415 , then the resulting 3-ball is LC, although it contains a double trefoil knot as a three-edge subcomplex.

Proposition 4.3. The removal of the tetrahedron 191415 from $S_{16,92}$ yields a locally constructible 3-ball $B_{16,91}$ with 16 vertices and 91 tetrahedra.

Proof. Let $D$ be the 2-ball given by the triangles 1915, 11415 and 91415 . Clearly $D$ is a subcomplex of the boundary of $B_{16,91}$; it is in fact equal to $\partial B_{16,91}$ minus the triangle 1914 . Our goal is to show that $B_{16,91}$ collapses onto $D$. The following is a certificate that this is true. 
First phase (pairs "triangle" $\rightarrow$ "tetrahedron"):

\begin{tabular}{|c|c|c|c|c|c|c|c|c|c|c|c|}
\hline 1914 & $\rightarrow$ & 18914 & 8914 & $\rightarrow$ & 28914 & 189 & $\rightarrow$ & 1789 & 289 & $\rightarrow 2$ & 2789 \\
\hline 178 & $\rightarrow$ & 1378 & 137 & $\rightarrow$ & 13711 , & 378 & $\rightarrow$ & 3578 & 138 & $\rightarrow$ & 13811 , \\
\hline 278 & $\rightarrow$ & 2478 & 2814 & $\rightarrow$ & 281214 & 1711 & $\rightarrow$ & 17911 & 357 & $\rightarrow$ & 3567 \\
\hline 567 & $\rightarrow$ & 4567 & 3811 & $\rightarrow$ & 35811 & 248 & $\rightarrow$ & 24816 & 3711 & $\rightarrow$ & 371114 , \\
\hline 279 & $\rightarrow$ & 27910 & 5811 & $\rightarrow$ & 81113 & 1811 & $\rightarrow$ & 181115 & 4816 & $\rightarrow$ & 45816 \\
\hline 1815 & $\rightarrow$ & 181015 & 2914 & $\rightarrow$ & 26914 & 81115 & $\rightarrow$ & 8111315 & 5816 & $\rightarrow$ & 581316 \\
\hline 51316 & $\rightarrow$ & 151316 & 269 & $\rightarrow$ & 26916 & 1911 & $\rightarrow$ & 191115 & 111315 & $\rightarrow$ & 9111315 , \\
\hline 1810 & $\rightarrow$ & 181014 & 6916 & $\rightarrow$ & 36916 & 247 & $\rightarrow$ & 24715 & 2415 & $\rightarrow 2$ & 23415 \\
\hline 11014 & $\rightarrow$ & 1101314 , & 457 & $\rightarrow$ & 578 & 2916 & $\rightarrow$ & 291016 , & & $\rightarrow$ : & 51114 \\
\hline 1513 & $\rightarrow$ & 1213 & 81014 & $\rightarrow$ & 01214 & 4516 & $\rightarrow$ & 14516 & 145 & $\rightarrow$ & 56 \\
\hline 356 & $\rightarrow$ & 35614 & 11314 & $\rightarrow$ & 131416 , & 1512 & $\rightarrow$ & 12512 & 71114 & $\rightarrow 7$ & 1214 , \\
\hline 91315 & $\rightarrow$ & 391315 & 3913 & $\rightarrow$ & 391213 & 156 & $\rightarrow$ & 1256 & 3714 & $\rightarrow:$ & 371416 , \\
\hline 71416 & $\rightarrow$ & 131416 , & 5614 & $\rightarrow$ & 614 & 234 & $\rightarrow$ & 23413 & 146 & $\rightarrow$ & 14612 \\
\hline 6713 & $\rightarrow$ & 671316 & 81015 & $\rightarrow$ & 8101215, & 467 & $\rightarrow$ & 46715 & 1416 & $\rightarrow$ & 141016 \\
\hline 51113 & $\rightarrow$ & 5111213 , & 4612 & $\rightarrow$ & 461215 & 21016 & $\rightarrow$ & 241016 & 111214 & $\rightarrow$ & 1214 \\
\hline 2512 & $\rightarrow$ & 251214 & 41215 & $\rightarrow$ & 341215 & 31213 & $\rightarrow$ & 341213 & 3916 & $\rightarrow:$ & 391216 , \\
\hline 31215 & $\rightarrow$ & 3121516 , & 3614 & $\rightarrow$ & 36914 & 101215 & $\rightarrow$ & 10121516 & 6716 & $\rightarrow$ & 36716 \\
\hline 01214 & $\rightarrow$ & 7101214 , & 11016 & $\rightarrow$ & 1101516 , & 1612 & $\rightarrow$ & 12612 & 71012 & $\rightarrow 7$ & 791012 , \\
\hline 71315 & $\rightarrow$ & 271315 & 2816 & $\rightarrow$ & 26816 & 91016 & $\rightarrow$ & 9101216 & 2413 & $\rightarrow 2$ & 241013 , \\
\hline 61316 & $\rightarrow$ & 681316 & 7912 & $\rightarrow$ & 791112 & 31315 & $\rightarrow$ & 231315 & 41213 & $\rightarrow 1$ & 141213 \\
\hline 41013 & $\rightarrow$ & 141013 & 6815 & $\rightarrow$ & 681215 & 2812 & $\rightarrow$ & 26812 & 3915 & $\rightarrow:$ & 391415 , \\
\hline 31416 & & 3141516 , & 11416 & & 141516 & & & & & & \\
\hline
\end{tabular}

Second phase (pairs "edge" $\rightarrow$ "triangle"):

\begin{tabular}{|c|c|c|c|c|c|c|c|c|c|c|c|c|c|c|}
\hline 89 & $\rightarrow$ & 789 & 29 & $\rightarrow$ & 2910 & 16 & $\rightarrow$ & 126 & 516 & $\rightarrow$ & 1516, & 38 & $\rightarrow$ & 358, \\
\hline 815 & $\rightarrow$ & 81215 & 13 & $\rightarrow$ & 1311 , & 1315 & $\rightarrow$ & 21315 & 15 & $\rightarrow$ & 125 & 17 & $\rightarrow$ & 179 \\
\hline 810 & $\rightarrow$ & 81012 & 18 & $\rightarrow$ & 1814 & 811 & $\rightarrow$ & 81113 & 28 & $\rightarrow$ & 268 & 35 & $\rightarrow$ & 3514, \\
\hline 1113 & $\rightarrow$ & 111213 & 12 & $\rightarrow$ & 1212 , & 814 & $\rightarrow$ & 81214 & 57 & $\rightarrow$ & 578 & 916 & $\rightarrow$ & 91216 , \\
\hline 613 & $\rightarrow$ & 6813 & 311 & $\rightarrow$ & 31114 & 812 & $\rightarrow$ & 6812 & 913 & $\rightarrow$ & 91213 , & 78 & $\rightarrow$ & 478 \\
\hline 111 & $\rightarrow$ & 11115 & 1114 & $\rightarrow$ & 51114 & 1115 & $\rightarrow$ & 91115 & 48 & $\rightarrow$ & 458 & 45 & $\rightarrow$ & 456 \\
\hline 511 & $\rightarrow$ & 51112 , & 56 & $\rightarrow$ & 256 & 47 & $\rightarrow$ & 4715 & 58 & $\rightarrow$ & 5813 & 25 & $\rightarrow$ & 14, \\
\hline 46 & $\rightarrow$ & 4615 & 513 & $\rightarrow$ & 51213 & 512 & $\rightarrow$ & 51214 & 68 & $\rightarrow$ & 6816 & 415 & $\rightarrow$ & 3415 \\
\hline 1213 & $\rightarrow$ & 11213 & 813 & $\rightarrow$ & 81316 & 112 & $\rightarrow$ & 1412 & 412 & $\rightarrow$ & 3412 , & 34 & $\rightarrow$ & 3413 , \\
\hline 313 & $\rightarrow$ & 2313 & 23 & $\rightarrow$ & 2315 & 215 & $\rightarrow$ & 2715 & 413 & $\rightarrow$ & 1413 & 715 & $\rightarrow$ & 15 \\
\hline 67 & $\rightarrow$ & 367 & 27 & $\rightarrow$ & 2713 & 615 & $\rightarrow$ & 61215 & 612 & $\rightarrow$ & 2612 & 37 & $\rightarrow$ & 16 \\
\hline 14 & $\rightarrow$ & 1410 & 1215 & $\rightarrow$ & 121516 & 716 & $\rightarrow$ & 71316 & 213 & $\rightarrow$ & 21013 & 212 & $\rightarrow$ & 21214 , \\
\hline 210 & $\rightarrow$ & 2410 & 214 & $\rightarrow$ & 2614 & 410 & $\rightarrow$ & 41016 & 24 & $\rightarrow$ & 2416 & 1214 & $\rightarrow$ & 71214 \\
\hline 26 & $\rightarrow$ & 2616 & 713 & $\rightarrow$ & 71314 & 616 & $\rightarrow$ & 3616 & 714 & $\rightarrow$ & 71014 & 614 & $\rightarrow$ & 14 \\
\hline 69 & $\rightarrow$ & 369 & 712 & $\rightarrow$ & 71112 & 710 & $\rightarrow$ & 7910 & 79 & $\rightarrow$ & 7911 & 911 & $\rightarrow$ & 91112 \\
\hline 1014 & $\rightarrow$ & 101314 , & 910 & $\rightarrow$ & 91012 , & 912 & $\rightarrow$ & 3912 & 1012 & $\rightarrow$ & 101216 , & 1216 & $\rightarrow$ & 31216 , \\
\hline 1314 & $\rightarrow$ & 131416 , & 1013 & $\rightarrow$ & 11013 & 39 & $\rightarrow$ & 3914 & 1416 & $\rightarrow$ & 141516 & 110 & $\rightarrow$ & 11015 \\
\hline 1316 & $\rightarrow$ & 11316 & 1016 & $\rightarrow$ & 101516 & 314 & $\rightarrow$ & 31415 & 315 & $\rightarrow$ & 31516 & 116 & $\rightarrow$ & 11516 . \\
\hline
\end{tabular}

Third phase (pairs "vertex" $\rightarrow$ "edge"):

$$
\begin{aligned}
13 & \rightarrow 113,5 \rightarrow 514,6 \rightarrow 36, \quad 10 \\
2 & \rightarrow 216,3 \rightarrow 1015, \quad 7 \rightarrow 711, \quad 11 \rightarrow 1112, \quad 12 \rightarrow 312,
\end{aligned}
$$


If we remove from the 3 -sphere $S_{16,92}$ the entire star of the vertex 1 (one of the three knot vertices), we obtain a 3 -ball $B_{15,66}$. By construction, $B_{15,66}$ contains a knotted spanning edge 23 , where the knot is the double trefoil. We proceed now to show the following properties:

(1) $B_{15,66}$ is not rectilinearly-embeddable in $\mathbb{R}^{3}$;

(2) $B_{15,66}$ is not collapsible;

(3) $B_{15,66}$ admits a discrete Morse function with one critical vertex, one critical edge and one critical triangle.

Proposition 4.4. $B_{15,66}$ is not rectilinearly-embeddable in $\mathbb{R}^{3}$.

Proof. The boundary of $B_{15,66}$ consists of the following 26 triangles:

$256, \quad 2512, \quad 2612, \quad 378, \quad 3711, \quad 3811, \quad 456, \quad 4516, \quad 4612$, $41013,41016,41213,51213,51316, \quad 789, \quad 7911, \quad 8914, \quad 81014$, $81015,81115,91115,91415,101314,101516,131416,141516$.

In particular, the five edges $25,513,1013,810$ and 38 form a boundary path from the vertex 2 to the vertex 3 . Together with the interior edge 23 , this path closes up to a hexagonal double trefoil knot. By Theorem 2.2, $B_{15,66}$ cannot be rectilinearly embedded in $\mathbb{R}^{3}$.

Theorem 4.5. $B_{15,66}$ admits a discrete Morse function with three critical faces, all of them belonging to the boundary $\partial B_{15,66}$.

Proof. We will show that there is a 2-dimensional subcomplex $C$ of $B_{15,66}$ such that:

- $B_{15,66}$ collapses onto $C$ and

- $C$ minus the triangle 258 collapses onto a pentagon.

Here is the right collapsing sequence:

First phase (pairs "triangle" $\rightarrow$ "tetrahedron"):

\begin{tabular}{|c|c|c|c|c|c|c|c|c|}
\hline 41016 & $\rightarrow 241016$ & 41013 & $\rightarrow 241013$ & 91415 & $\rightarrow 391415$ & 101516 & $\rightarrow$ & 10121516 \\
\hline 81115 & $\rightarrow 8111315$ & 3811 & $\rightarrow 35811$ & 81315 & $\rightarrow 681315$ & 131416 & $\rightarrow$ & 7131416 , \\
\hline 4516 & $\rightarrow \quad 45816$ & 6815 & $\rightarrow 681215$ & 456 & $\rightarrow 4567$ & 81015 & $\rightarrow$ & 8101215 , \\
\hline 8914 & $\rightarrow 28914$ & 2413 & $\rightarrow 23413$ & 141516 & $\rightarrow 3141516$ & 2512 & $\rightarrow$ & 251214 \\
\hline 4816 & $\rightarrow 24816$ & 2814 & $\rightarrow 281214$ & 248 & $\rightarrow 2478$ & 81012 & $\rightarrow$ & 8101214 , \\
\hline 2313 & $\rightarrow 231315$ & 3711 & $\rightarrow 371114$ & 4612 & 461215 , & 2612 & $\rightarrow$ & 26812 \\
\hline 91115 & $\rightarrow 9111315$ & 2816 & $\rightarrow 26816$ & 41215 & $\rightarrow 341215$ & 289 & $\rightarrow$ & 2789 \\
\hline 31416 & $\rightarrow 371416$ & 458 & $\rightarrow 4578$ & 567 & $\rightarrow 3567$ & 356 & $\rightarrow$ & 35614 \\
\hline 6813 & $\rightarrow \quad 681316$ & 31315 & $\rightarrow 391315$ & 3413 & 341213 , & 5816 & $\rightarrow$ & 581316 , \\
\hline 247 & $\rightarrow 24715$ & 51214 & $\rightarrow 5111214$ & 357 & $\rightarrow 3578$ & 2616 & $\rightarrow$ & 26916 \\
\hline 21516 & $\rightarrow 3121516$ & 2415 & $\rightarrow 23415$ & 61316 & $\rightarrow 671316$ & 2914 & $\rightarrow$ & 26914 \\
\hline 2916 & $\rightarrow 291016$ & 2614 & $\rightarrow 25614$ & 51213 & $\rightarrow 5111213$ & 31216 & $\rightarrow$ & 391216 , \\
\hline 71314 & $\rightarrow 7101314$ & 31114 & $\rightarrow 351114$ & 71114 & $\rightarrow 7111214$ & 51113 & $\rightarrow$ & 581113 \\
\hline 91216 & $\rightarrow 9101216$ & 71013 & $\rightarrow 271013$ & 91012 & $\rightarrow 791012$ & 7910 & $\rightarrow$ & 27910 \\
\hline 3614 & $\rightarrow 36914$ & 3916 & $\rightarrow 36916$ & 367 & $\rightarrow 36716$ & 4715 & $\rightarrow$ & 46715 \\
\hline 31213 & $\rightarrow 391213$ & 71012 & $\rightarrow 7101214$ & 21315 & $\rightarrow 271315$ & 7912 & $\rightarrow$ & 791112 , \\
\hline 91112 & $\rightarrow 9111213$ & 6715 & $\rightarrow 671315$ & & & & & \\
\hline
\end{tabular}


Second phase (pairs "edge" $\rightarrow$ "triangle"):

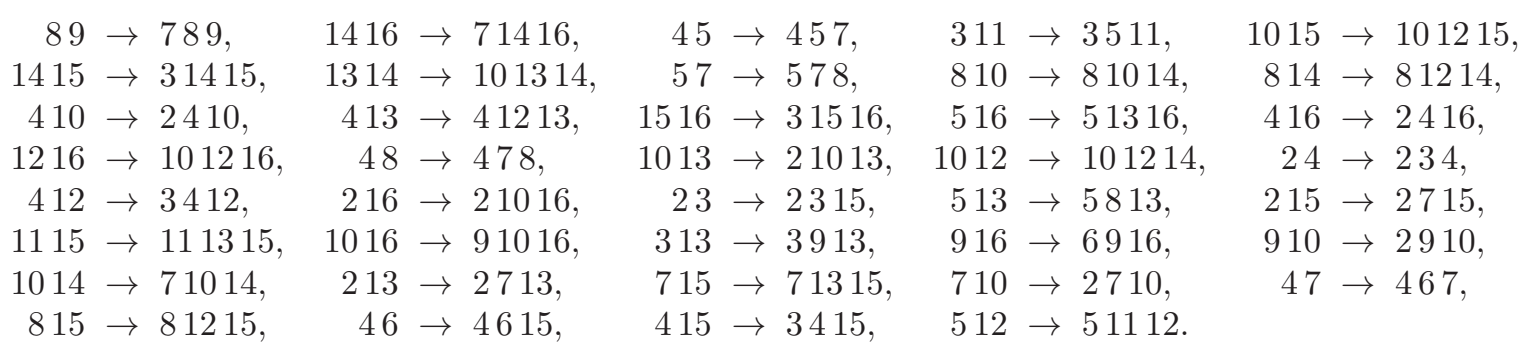

Let $C$ be the obtained 2-complex. Note that $C$ contains the triangle 258 , which belongs to $\partial B_{15,66}$ and has not been collapsed yet. Let $D$ be the complex obtained from $C$ after removing the (interior of the) triangle 258 . Here is a proof:

First phase (pairs "edge" $\rightarrow$ "triangle"):

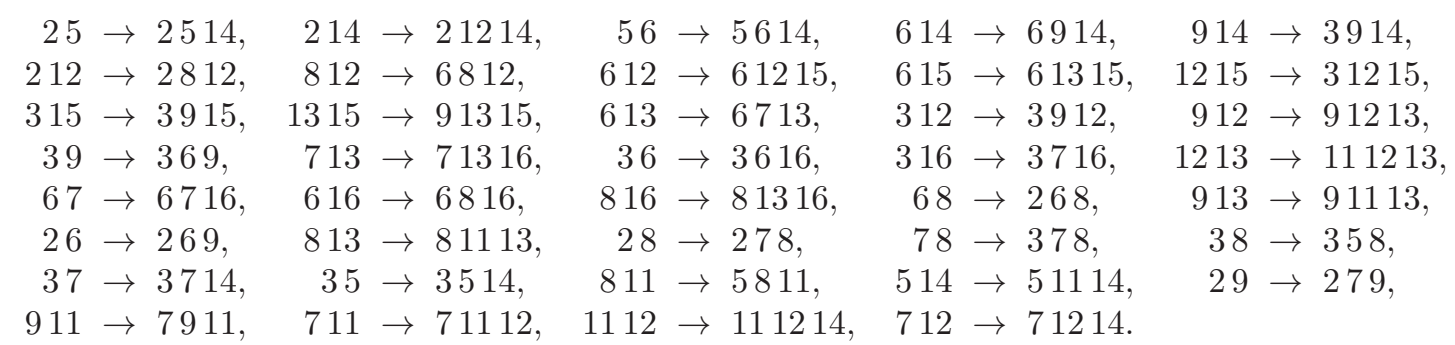

Final phase (pairs "vertex" $\rightarrow$ "edge"):

$2 \rightarrow 27,15 \rightarrow 915,3 \rightarrow 314,12 \rightarrow 1214,6 \rightarrow 69,8 \rightarrow 58,5 \rightarrow 511,9 \rightarrow 79$.

At this point we are left with the pentagon $P$ given by the five edges $714,716,1113$, 1114 , and 1316 . The latter edge, 1316 , belongs to the boundary of $B_{15,66}$. Clearly, $P$ minus this edge yields a collapsible 1 -ball. Thus, $B_{15,66}$ admits a discrete Morse function whose critical faces are the vertex 13, the edge 1366 and the triangle 258 . This discrete Morse function is the best possible, since $B_{15,66}$ cannot be collapsible (because of its knotted spanning edge 23 ).

\section{$5 \quad$ The triple trefoil}

In this section, we are constructing a triangulation $S_{44,284}$ of the 3 -sphere $S^{3}$ that contains a triple trefoil knot with three edges in its 1-skeleton. We then use bistellar flips to obtain a reduced triangulation $S_{18,125}$.

As before for the double trefoil, we place a triple trefoil knot on the three edges 12 , 23,13 in $\mathbb{R}^{3}$, as depicted in Figure 4. Each of the three knot edges is protected by a spindle; see Figure 5 for the spindles and Table 7 for the list of tetrahedra of the spindles.

To close the holes of the knot we glue in the membrane triangles of Table 8 and then add the local cones with respect to the vertices $34,35, \ldots, 43$ from Table 9 to obtain a 3-ball $B_{43,214}$.

Finally, we add to $B_{43,214}$ the cone over its boundary with respect to the vertex 44 (as given in Table 10) to obtain the 3 -sphere $S_{44,284}$. 


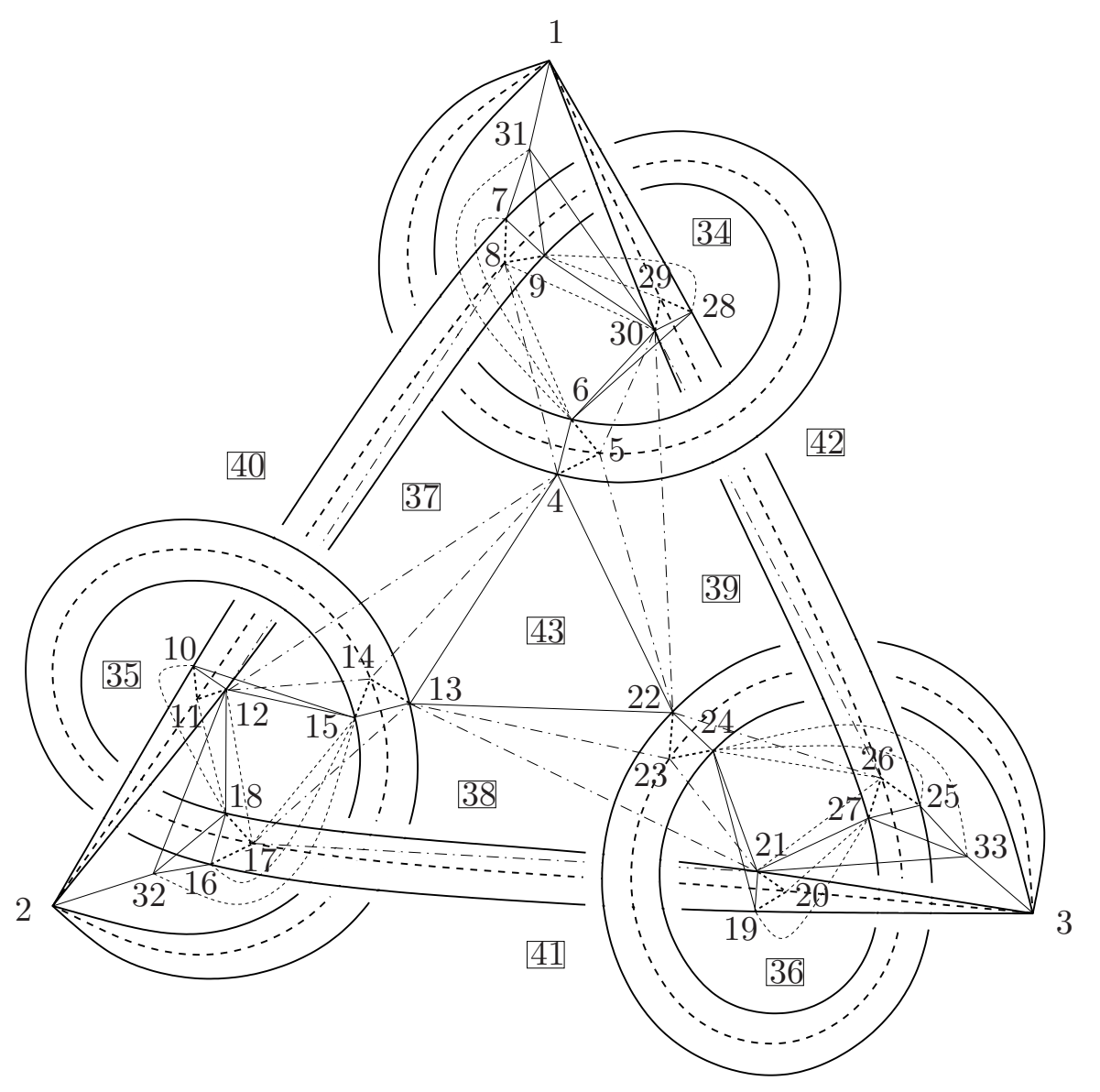

Figure 4: The triple trefoil in the sphere $S_{44,284}$.

Proposition 5.1. The 3 -sphere $S_{44,284}$ consists of 284 tetrahedra and 44 vertices. It has face vector $f=(44,328,568,284)$ and contains the triple trefoil knot on three edges in its 1-skeleton.

Again, the 3 -sphere $S_{44,284}$ is not minimal with the property of containing the triple trefoil knot in its 1-skeleton. The smallest triangulation we found via bistellar flips is $S_{18,125}$; see Table 11 for the list of facets of $S_{18,125}$.

Theorem 5.2. The 3-sphere $S_{18,125}$ consists of 125 tetrahedra and 18 vertices. It has face vector $f=(18,143,250,125)$ and contains the triple trefoil knot on three edges in its 1-skeleton.

Because of the knot, $S_{18,125}$ is not LC. So it cannot admit a discrete Morse with fewer than four critical cells. However, it does admit a discrete Morse function with one critical vertex, one critical edge, one critical triangle and one critical tetrahedron, as we once more found by a random search.

Theorem 5.3. $S_{18,125}$ admits a discrete Morse function with one critical vertex, one critical edge, one critical triangle and one critical tetrahedron. 

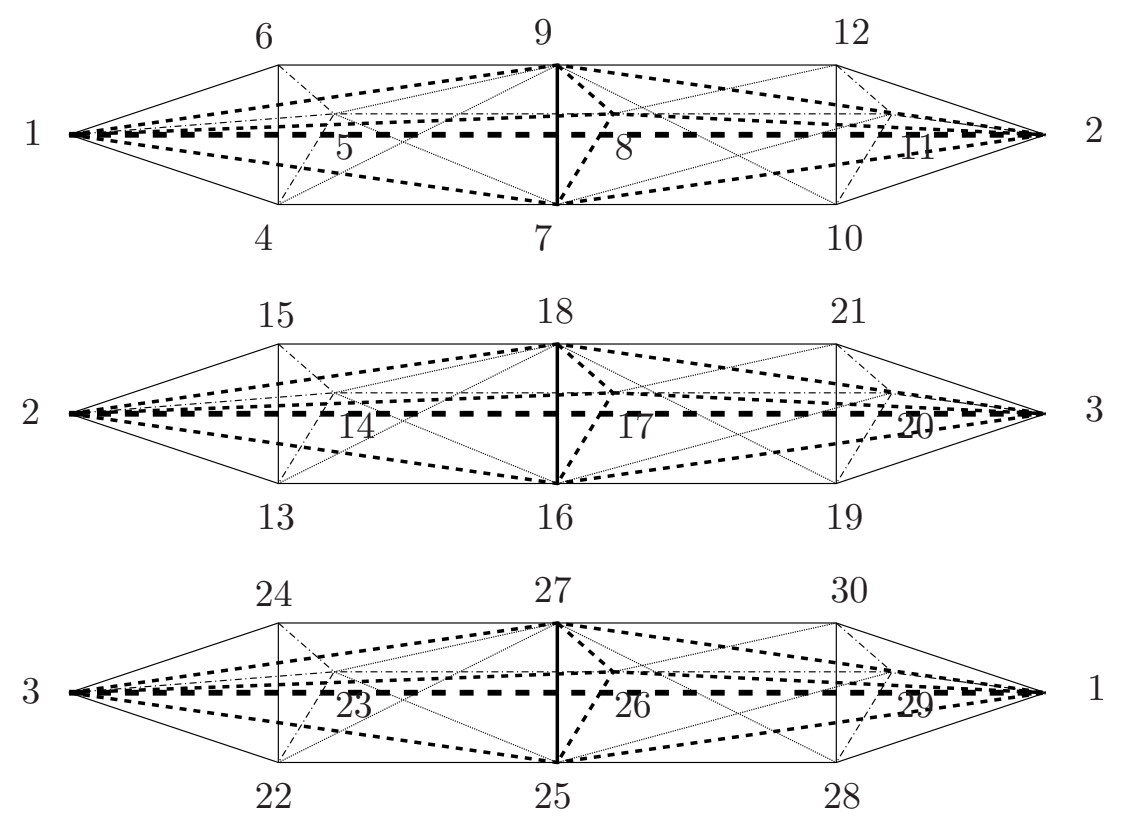

Figure 5: The spindles of $S_{44,284}$.

Table 7: Part A of the sphere $S_{44,284}$ : The three spindles.

\begin{tabular}{lllllllll}
\hline 1278 & 1469 & 27910 & 231617 & 2131518 & 3161819 & 132526 & 3222427 & 1252728 \\
1279 & 1479 & 291012 & 231618 & 2131618 & 3181921 & 132527 & 3222527 & 1272830 \\
1289 & 1457 & 27811 & 231718 & 2131416 & 3161720 & 132627 & 3222325 & 1252629 \\
& 1578 & 271011 & & 2141617 & 3161920 & & 3232526 & 1252829 \\
& 1569 & 28912 & & 2141518 & 3171821 & & 3232427 & 1262730 \\
& 1589 & 281112 & & 2141718 & 3172021 & & 3232627 & 1262930 \\
\hline
\end{tabular}

Table 8: The triangles of the membranes in the sphere $S_{44,284}$.

\begin{tabular}{lllllllll}
\hline \multicolumn{7}{c}{41322} & & \\
8930 & 62830 & 13031 & 121718 & 101215 & 21232 & 212627 & 192124 & 32133 \\
6830 & 6928 & 93031 & 121517 & 101518 & 121832 & 212426 & 192427 & 212733 \\
468 & 92829 & 7931 & 131517 & 101118 & 161832 & 222426 & 192027 & 252733 \\
4812 & 92930 & 1631 & 131721 & 111218 & 21532 & 222630 & 202127 & 32433 \\
41214 & & 6731 & 132123 & & 151632 & 52230 & & 242533 \\
41314 & & 678 & 132223 & & 151617 & 4522 & & 242526 \\
121415 & & 212324 & & & 5630 & & \\
\hline
\end{tabular}


Table 9: Part B of the sphere $S_{44,284}$ : Tetrahedra to thicken Part A to a ball $B_{43,214}$.

\begin{tabular}{|c|c|c|c|c|c|}
\hline 9293031 & 11121832 & 20212733 & 1313740 & 2323841 & 3333942 \\
\hline 1293031 & 2111232 & 3202133 & 7313740 & 16323841 & 25333942 \\
\hline 793134 & 16183235 & 25273336 & 7103740 & 16193841 & 25283942 \\
\hline 9293134 & 11183235 & 20273336 & 10153740 & 19243841 & 6283942 \\
\hline 1293134 & 2113235 & 3203336 & 14153740 & 23243841 & 563942 \\
\hline 1282934 & 2101135 & 3192036 & 14173740 & 23263841 & 583942 \\
\hline 46934 & 13151835 & 22242736 & 14151840 & 23242741 & 56942 \\
\hline 47934 & 13161835 & 22252736 & 14171840 & 23262741 & 58942 \\
\hline 9282934 & 10111835 & 19202736 & 10151840 & 19242741 & 692842 \\
\hline 692834 & 10151835 & 19242736 & 10111840 & 19202741 & 9282942 \\
\hline 6283034 & 10121535 & 19212436 & 11121840 & 20212741 & 9293042 \\
\hline \multirow[t]{2}{*}{1283034} & 2101235 & 3192136 & 8111240 & 17202141 & 26293042 \\
\hline & & & 781140 & 16172041 & 25262942 \\
\hline 473439 & 13163537 & 22253638 & 7101140 & 16192041 & 25282942 \\
\hline \multirow[t]{2}{*}{4343739} & 13353738 & 22363839 & 67840 & 15161741 & 24252642 \\
\hline & & & 673140 & 15163241 & 24253342 \\
\hline 10121415 & 19212324 & 562830 & 163140 & 2153241 & 3243342 \\
\hline 791037 & 16181938 & 25272839 & 14640 & 2131541 & 3222442 \\
\hline 9101237 & 18192138 & 27283039 & 46840 & 13151741 & 22242642 \\
\hline 10121437 & 19212338 & 5283039 & 481240 & 13172141 & 22263042 \\
\hline 10141537 & 19232438 & 562839 & & & \\
\hline 13141637 & 22232538 & 45739 & & 4132243 & \\
\hline 14161737 & 23252638 & 57839 & & 4133743 & \\
\hline 4131437 & 13222338 & 452239 & & 13373843 & \\
\hline 4121437 & 13212338 & 5223039 & & 13223843 & \\
\hline 481237 & 13172138 & 22263039 & & 22383943 & \\
\hline 891237 & 17182138 & 26273039 & & 4223943 & \\
\hline 46837 & 13151738 & 22242639 & & 4373943 & \\
\hline 683037 & 12151738 & 21242639 & & & \\
\hline 893037 & 12171838 & 21262739 & & & \\
\hline 9303137 & 12183238 & 21273339 & & & \\
\hline 1303137 & 2123238 & 3213339 & & & \\
\hline 793137 & 16183238 & 25273339 & & & \\
\hline 1303437 & 2123538 & 3213639 & & & \\
\hline 6303437 & 12153538 & 21243639 & & & \\
\hline 463437 & 13153538 & 22243639 & & & \\
\hline
\end{tabular}


Table 10: Part C of the sphere $S_{44,284}$ : Cone over the boundary of the ball $B_{43,214}$.

\begin{tabular}{lllllll}
\hline 14544 & 144044 & 15644 & 163144 & 1313444 & 1343744 & 1374044 \\
2131444 & 2134144 & 2141544 & 2153244 & 2323544 & 2353844 & 2384144 \\
3222344 & 3224244 & 3232444 & 3243344 & 3333644 & 3363944 & 3394244 \\
452244 & 4121444 & 4124044 & 4131444 & 4132244 & 563044 & 5223044 \\
67844 & 673144 & 683044 & 783944 & 7313444 & 7343944 & 893044 \\
894244 & 8394244 & 9304244 & 12141544 & 12151744 & 12171844 & 12184044 \\
13212344 & 13214144 & 13222344 & 15161744 & 15163244 & 16173744 & 16323544 \\
16353744 & 17184044 & 17374044 & 21232444 & 21242644 & 21262744 & 21274144 \\
22304244 & 24252644 & 24253344 & 25263844 & 25333644 & 25363844 & 26274144 \\
26384144 & 34373944 & 35373844 & 36383944 & 37384344 & 37394344 & 38394344 \\
\hline
\end{tabular}

Table 11: The sphere $S_{18,125}$.

\begin{tabular}{llllllll}
\hline 1249 & 12415 & 12915 & 13810 & 13812 & 131012 & 14514 & 14516 \\
14914 & 141516 & 15711 & 15714 & 151117 & 151216 & 151217 & 17112 \\
171216 & 171416 & 181013 & 181217 & 181318 & 181718 & 191415 & 1101213 \\
1111218 & 111718 & 1121318 & 1141516 & 23513 & 23514 & 231314 & 24615 \\
24617 & 24917 & 251014 & 251018 & 251318 & 261112 & 261116 & 261215 \\
261617 & 27810 & 27811 & 271018 & 271112 & 271216 & 271618 & 281013 \\
281116 & 281318 & 281618 & 291215 & 291216 & 291617 & 2101314 & 34812 \\
34815 & 341012 & 341016 & 341516 & 35713 & 35714 & 36914 & 36918 \\
361116 & 361118 & 361417 & 361617 & 37913 & 37918 & 371418 & 381015 \\
391314 & 3101517 & 3101617 & 3111516 & 3111517 & 3111718 & 3141718 & 451014 \\
451016 & 46815 & 46817 & 481217 & 491314 & 491317 & 4101213 & 4101314 \\
4121317 & 5678 & 56713 & 5689 & 56918 & 561318 & 57811 & 58911 \\
591016 & 591018 & 591115 & 591215 & 591216 & 5111517 & 5121517 & 67815 \\
671315 & 68914 & 681417 & 6111218 & 6121315 & 6121318 & 781015 & 791017 \\
791018 & 791317 & 7101517 & 7131517 & 7141618 & 891114 & 8111416 & 8141618 \\
8141718 & 9101617 & 9111415 & 11141516 & 12131517 & & & \\
\hline
\end{tabular}

Similar to before, by deleting the vertex 1 from $S_{18,125}$, we obtain a 3-ball $B_{17,95}$ with the following properties:

(1) $B_{17,95}$ contains a knotted spanning edge 23 , where the knot is the triple trefoil;

(2) $B_{17,95}$ is not rectilinearly-embeddable in $\mathbb{R}^{3}$;

(3) $B_{17,95}$ is not collapsible;

(4) $B_{17,95}$ admits a discrete Morse function with one critical vertex, two critical edges and two critical triangles (found with the randomized approach of [10]), and this is best possible. 


\section{Non-evasiveness and vertex-decomposability}

In this section, we show that all vertex-decomposable balls are non-evasive, while the converse is false already in dimension three. For example, we show that Rudin's ball is non-evasive, but it is neither vertex-decomposable nor shellable. The following Lemma is well known.

Lemma 6.1. Let $v$ be a shedding vertex of a vertex-decomposable d-ball B. Then $v$ lies on the boundary of the ball. In particular,

(i) $\operatorname{link}(v, B)$ is a vertex-decomposable $(d-1)$-ball;

(ii) $\operatorname{del}(v, B)$ is a vertex-decomposable d-ball.

Proof idea: If $v$ is an interior vertex, then the deletion of $v$ is $d$-dimensional but not $(d-1)$-connected and therefore not vertex-decomposable.

Theorem 6.2. Every vertex-decomposable d-ball is non-evasive. In particular, all 2-balls are non-evasive.

Proof. A zero-dimensional vertex-decomposable ball is just a point, so it is indeed nonevasive. Let $B$ be a vertex-decomposable $d$-ball, with $d>0$. By Lemma 6.1 there is a boundary vertex $v \operatorname{such}$ that $\operatorname{del}(v, B)$ is a vertex-decomposable $d$-ball and $\operatorname{link}(v, B)$ is a vertex-decomposable $(d-1)$-ball. The deletion of $v$ from $B$ has fewer facets than $B$, and the link of $v$ in $B$ has smaller dimension than $B$. By double induction on the dimension and the number of facets, we may assume that both $\operatorname{del}(v, B)$ and $\operatorname{link}(v, B)$ are nonevasive. By definition, then, $B$ is non-evasive.

Next, we prove that the converse of Theorem 6.2 above is false.

Theorem 6.3. Rudin's ball $R$, which has 14 vertices and 41 facets, is non-evasive.

Proof. Rudin's ball is given by the following 41 facets [32]:

$\begin{array}{lllllll}13713, & 13913, & 15711, & 15911, & 171113, & 191113, & 24814, \\ 241014, & 26812, & 261012, & 281214, & 2101214, & 34711, & 34712, \\ 361011, & 361014, & 371213, & 371114, & 391213, & 3101114, & 45812, \\ 45813, & 471112, & 481112, & 481314, & 4101314, & 56913, & 56914, \\ 571114, & 581213, & 591213, & 591114, & 681112, & 691314, & 6101112, \\ 6101314, & 7111213, & 8121314, & 9111314, & 10111214, & 11121314 . & \end{array}$

To prove non-evasiveness, we claim that the sequence

$$
\left(a_{1}, \ldots, a_{14}\right)=(3,4,5,12,13,1,7,9,14,8,11,10,2,6)
$$

has the following two properties:

(I) For each $i \leqslant 5, \operatorname{link}_{a_{i}} \operatorname{del}_{a_{1}, \ldots, a_{i-1}} R$ is a non-evasive 2-complex;

(II) $\operatorname{del}_{3,4,5,12,13} R$ is a non-evasive 2-complex.

To prove that an arbitrary 2-complex $C$ with $n$ vertices is non-evasive, we need to find an order $a_{1}, \ldots, a_{k}, a_{k+1}, \ldots, a_{n}$ of its vertices so that: 
(i) For each $i \leqslant k$, link $\operatorname{ael}_{a_{i}} \operatorname{del}_{a_{1}, \ldots, a_{i-1}} R$ is a tree;

(ii) $\operatorname{del}_{a_{1}, \ldots, a_{k}} R$ is a tree.

All trees and all simplicial 2-balls are vertex-decomposable and non-evasive, cf. Theorem 6.2. In particular, the link of 3 in $R$ is a non-evasive 2-ball. Let us delete this vertex 3 , and proceed with the proof of the claim:

- The link of 4 in $\operatorname{del}_{3} R$ is the 2 -complex $C$ given by the following 8 facets

$$
2814,21014,5812,71112,81112,81314, \quad 101314,5813 .
$$

Let us show that $C$ is non-evasive. The link of 7 in $C$ is a single edge, hence nonevasive. The deletion of 7 from $C$ yields a complex with the same triangles as $C$, except 71112 . Inside this smaller complex, the link of 8 is a path, and the deletion of 8 yields the 2 -complex

$$
21014, \quad 512, \quad 1112, \quad 1314, \quad 101314,513 .
$$

This is a 2-ball with a 3 -edge path attached, hence non-evasive. In particular, $C$ is non-evasive.

- The link of 5 in $\operatorname{del}_{3,4} R$ is the 2 -complex $D$ given by the following 8 facets

$$
1711,1911,6913,6914,71114,81213,9111491213 .
$$

We can delete 8 first (its link is an edge), then 9 (because its link is a 6-edge path). The resulting 2-complex,

$$
1711,613,614,71114, \quad 1213
$$

is a 2-ball with a 3 -edge path attached, hence non-evasive. So $D$ is also non-evasive.

- The link of 12 in $\operatorname{del}_{3,4,5} R$ is the (non-pure) 2-complex $E$ given by the following 11 facets

$268, \quad 2610, \quad 2814,21014,6811,61011,71113,81314,913$, 101114,111314 .

We can delete 9 and 7 , as their links are a point and an edge (respectively); after that, we delete 13, whose link is now a path. The resulting 2-complex $E^{\prime}$ has 7 facets:

$$
268,2610,2814,21014,6811,61011,101114 .
$$

The link of 14 inside $E^{\prime}$ is a 3-edge path, and the deletion of 14 from $E^{\prime}$ yields a (non-evasive) 2-ball. So, $E^{\prime}$ and $E$ are non-evasive.

- The link of 13 in $\operatorname{del}_{3,4,5,12} R$ is the 2-complex $F$ given by the following 6 facets

$$
\text { 1711, 1911, 6914, 61014, 814, } 91114 .
$$

We can delete 8 first (its link is a point), then 7 (its link is single edge). The resulting 2-complex is a 2-ball. In particular, $F$ is non-evasive. 
- Finally, let us examine the 2-complex $G:=\operatorname{del}_{3,4,5,12,13} R$. It consists of 13 facets: $1711, \quad 1911, \quad 268, \quad 2610, \quad 21014, \quad 2814,6811,6914,61011$, $61014, \quad 71114, \quad 91114, \quad 101114$.

From $G$ we can delete 1 (it has a 2-edge link), then 7 (1-edge link), and then 9 (2-edge link). The resulting 2-complex $H:=\operatorname{del}_{1,7,9} G$ consists of 8 facets:

$268,2610,21014,2814,6811,61011,61014, \quad 1011,14$.

The link of 14 inside $H$ is a 4-edge path, and the deletion from $H$ of 14 yields a 2-ball. So $H$ is non-evasive; therefore $G$ is non-evasive as well.

Corollary 6.4. Some non-evasive balls are (constructible and) not shellable.

For a more general statement on non-evasiveness of convex 3-balls see [2].

Proposition 6.5. Let $B_{7,10}$ be the smallest shellable 3 -ball that is not vertex-decomposable [27]. This $B_{7,10}$ is non-evasive.

Proof. $B_{7,10}$ is given by the following 10 tetrahedra:

$0126,0134,0136,0235,0256,0356, \quad 1245, \quad 1246, \quad 1346,2456$.

As explained in [27], the deletion of 6 yields the (non-pure!) 3-complex $A$ given by the facets

$$
012, \quad 0134, \quad 0235, \quad 1245 \text {. }
$$

The link of the vertex 5 in $A$ consists of two triangles with a point in common; this is non-evasive. Deleting 5 from $A$, we obtain the 3 -complex $B$ with the following facets.

$$
012, \quad 0134, \quad 023,124 .
$$

The link of the vertex 4 inside $B$ is a triangle with an edge attached, hence non-evasive. The deletion of the vertex 4 from $B$ is a 2-ball. Therefore, $B$ is non-evasive, $A$ is nonevasive, and $B_{7,10}$ is non-evasive as well. The sequence of deletions certificating its nonevasiveness is the 'countdown sequence' $6-5-4-3-2-1-0$.

Corollary 6.6. Some non-evasive balls are shellable but not vertex-decomposable.

Proposition 6.7. Let $B_{9,18}$ be the smallest non-shellable 3-ball, described in [26]. $B_{9,18}$ is non-evasive and constructible.

Proof. $B_{9,18}$ is given by the following 18 tetrahedra:

$0123,0124,0145,0157,0168,0178, \quad 0234, \quad 0678, \quad 1236$, $1245,1258,1268,1578,2347,2367,2467,2468,4678$. 
Consider the 2-sphere $S$ given by the following 12 triangles:

$$
023, \quad 024,036,045, \quad 057,068, \quad 078, \quad 236,245, \quad 258, \quad 268,578 .
$$

It is easy to see that $S$ minus the triangle 036 is the same 2-complex as the link of 1 inside $B_{9,18}$. Since a 2 -sphere minus a triangle yields a 2-ball, and all 2-balls are shellable, it follows that the link of 1 inside $B_{9,18}$ is shellable. Since shellability is preserved by taking cones, the closed star $C_{1}$ of 1 inside $B_{9,18}$ is also shellable. Let $B_{1}:=C_{1} \cup 0678$. Since $C_{1} \cap 0678$ consists of the two triangles 068 and $078, B_{1}$ is also shellable. (A shelling order for $B_{1}$ is the shelling order for $C_{1}$, plus 0678 as last facet.) Now, let $B_{2}$ be the shellable 3 -ball with 7 vertices (labeled by $0,2,3,4,6,7,8$ ) with the following 6 facets, already given in a possible shelling order:

$$
0234,2347,2367,2467,2468,4678 .
$$

Clearly, $B_{9,18}$ splits as $B_{1} \cup B_{2}$. Moreover, the intersection $B_{1} \cap B_{2}$ is a 2-ball, given by the following 5 facets:

$$
023, \quad 024, \quad 236,268,678 .
$$

In particular, $B_{9,18}$ is constructible. We still have to prove that $B$ is non-evasive; we will show this by deleting the vertices $1-0-6-3-7-2-4-5-8$, in this order. The link of vertex 1 in $B_{9,18}$ is the (non-evasive, shellable) 2-ball descrived above. The deletion of 1 from $B_{9,18}$ yields the following 3-complex $A$ :

$$
0234, \quad 0678, \quad 2347,2367,2467,2468, \quad 4678, \quad 045, \quad 057, \quad 245, \quad 258, \quad 578 .
$$

Inside $A$, the link of the vertex 0 consist of two triangles joined by a 2-edge path. Such a 2 -complex is clearly non-evasive. Deleting the vertex 0 from $A$ we obtain the 3 -complex $B$ described as follows:

$$
2347,2367,2467,2468,4678,245,258,578 .
$$

Next, we delete 6 , whose link inside $B$ is a 2-ball with 4 triangles. The result is this 3-complex $C$ :

$$
2347,245,248,258, \quad 478, \quad 578 .
$$

From $C$ we can delete first 3 (whose link is a triangle) and then 7 (whose link is a 3 -edge path). The result is a 2-ball, so $C$ is non-evasive. As a consequence, $B, A$ and $B_{9,18}$ are all non-evasive.

Our last result highlights the positive effects of barycentric subdivisions.

Proposition 6.8. Let $B$ be a simplicial complex.

(i) Although $B_{9,18}$ is not shellable, its barycentric subdivision is vertex-decomposable.

(ii) Although $S_{13,56}$ is not constructible, its barycentric subdivision is vertex-decomposable.

(iii) Although $B_{12,38}$ is evasive and not $L C$, its barycentric subdivision is $L C$ and nonevasive. 
Proof. Sequences of deletions that prove vertex-decomposability of sd $B_{9,18}$ and $S_{13,56}$ were found with a computer backtrack search. Since $B_{12,38}$ is collapsible, by a result of Welker sd $B_{12,38}$ is non-evasive [33]. Since $B_{12,38}$ is a collapsible 3-ball, by a result of the first author sd $B_{12,38}$ is locally constructible [8].

Corollary 6.9. Some non-evasive balls are (LC and) not constructible.

Proof. The barycentric subdivision of $B_{12,38}$ cannot be constructible by Theorem 2.1, because it contains a knotted spanning arc of two edges.

\section{Open problems}

The following questions remain open:

- Are there constructible $d$-spheres that are not shellable? The problem is open already for $d=3$.

- Are there non-evasive balls with a knotted spanning edge?

- Are there examples of non-shellable spheres that become vertex-decomposable after stacking all facets? (This would imply that a non-simplicial 4-ball can be vertexdecomposable but not shellable.)

- Are there evasive collapsible 4-balls?

- Are there non-evasive balls that are not LC? Are there LC (3-)balls that are evasive?

- Are the 3 -spheres $S_{16,92}$ and $S_{18,125}$ vertex-minimal with the property of having the double trefoil and the triple trefoil knot on three edges in their 1-skeleton, respectively? What happens if we replace the square knot by the granny knot?

\section{Acknowledgements.}

Thanks to Jonathan Barmak and Alex Engström for helpful discussions.

\section{References}

[1] C. C. Adams, B. M. Brennan, D. L. Greilsheimer, and A. K. Woo. Stick numbers and composition of knots and links. J. Knot Theory Ramifications, 6:149-161, 1997.

[2] K. Adiprasito and B. Benedetti. Tight complexes in 3-space admit perfect discrete Morse functions. Preprint (2012, 13 pages) available at arxiv:1202.3390.

[3] J. Ambjørn, B. Durhuus, and T. Jonsson. Quantum Geometry. Cambridge University Press, Cambridge, 1997.

[4] J. Ambjørn and S. Varsted. Three dimensional simplicial quantum gravity. Nucl. Phys. B, 373:557-577, 1992.

[5] B. Bagchi and B. Datta. On stellated spheres, shellable balls, lower bounds and a combinatorial criterion for tightness. Preprint (2012, 46 pages) available at arxiv:1102.0856v2. 
[6] J. A. Barmak and G. E. Minian. Strong homotopy types, nerves and collapses. Discrete Comput. Geometry, 47:301-328, 2012.

[7] B. Benedetti. On Locally Constructible Manifolds. $\mathrm{PhD}$ thesis, TU Berlin (2010). Available online at http://opus4.kobv.de/opus4-tuberlin/frontdoor/index/index/docId/2388.

[8] B. Benedetti. Discrete Morse theory for manifolds with boundary. Trans. Amer. Math. Soc., 364:6631-6670, 2012.

[9] B. Benedetti and F. H. Lutz. The dunce hat and a minimal non-extendably collapsible 3-ball. Preprint (2013, 6 pages) available at arxiv:0912.3723v2, to appear in Electronic Geometry Models.

[10] B. Benedetti and F. H. Lutz. Random discrete Morse theory and a new library of triangulations. Preprint (2013, 33 pages) available at arxiv:1303.6422.

[11] B. Benedetti and G. M. Ziegler. On locally constructible spheres and balls. Acta Math., 206:205-243, 2011.

[12] R. H. Bing. Some aspects of the topology of 3-manifolds related to the Poincaré conjecture. In Lectures on Modern Mathematics, vol. II (T. Saaty, ed.), pages 93 128. Wiley, New York, 1964.

[13] A. Björner and F. H. Lutz. Simplicial manifolds, bistellar flips and a 16-vertex triangulation of the Poincaré homology 3-sphere. Experiment. Math., 9:275-289, 2000.

[14] R. Dougherty, V. Faber, and M. Murphy. Unflippable tetrahedral complexes. Discrete Comput. Geom., 32:309-315, 2004.

[15] B. Durhuus and T. Jonsson. Remarks on the entropy of 3-manifolds. Nucl. Phys. B, 445:182-192, 1995.

[16] R. Ehrenborg and M. Hachimori. Non-constructible complexes and the bridge index. European J. Combin., 22:475-491, 2001.

[17] A. Engström. Discrete Morse functions from Fourier transforms. Experiment. Math., 18:45-53, 2009.

[18] R. Forman. Morse theory for cell complexes. Adv. in Math., 134:90-145, 1998.

[19] R. Goodrick. Non-simplicially collapsible triangulations of $I^{n}$. Proc. Camb. Phil. Soc., 64:31-36, 1968.

[20] M. Hachimori and K. Shimokawa. Tangle sum and constructible spheres. J. Knot Theory Ramifications, 13:373-383, 2004.

[21] M. Hachimori and G. M. Ziegler. Decompositions of simplicial balls and spheres with knots consisting of few edges. Math. Z., 235:159-171, 2000.

[22] M.-E. Hamstrom and R. P. Jerrard. Collapsing a triangulation of a "knotted" cell. Proc. Amer. Math. Soc., 21:327-331, 1969.

[23] J. Kahn, M. Saks, and D. Sturtevant. A topological approach to evasiveness. Combinatorica, 4:297-306, 1984. 
[24] W. B. R. Lickorish. Unshellable triangulations of spheres, European J. Combin., 12:527-530, 1991.

[25] W. B. R. Lickorish and J. M. Martin. Triangulations of the 3-ball with knotted spanning 1-simplexes and collapsible $r$-th derived subdivisions. Trans. Amer. Math. Soc., 170:451-458, 1972.

[26] F. H. Lutz. A vertex-minimal non-shellable simplicial 3-ball with 9 vertices and 18 facets. Electronic Geometry Models, No. 2003.05.004, 2004.

[27] F. H. Lutz. Vertex-minimal not vertex-decomposable balls. Electronic Geometry Models, No. 2003.06.001, 2004.

[28] F. H. Lutz. BISTELLAR, Version 11/03.

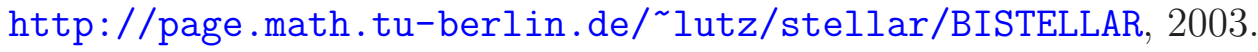

[29] F. H. Lutz. Small examples of nonconstructible simplicial balls and spheres. SIAM J. Discrete Math., 18:103-109, 2004.

[30] U. Pachner. P. L. homeomorphic manifolds are equivalent by elementary shellings. European J. Combin., 12:129-145, 1991.

[31] J. S. Provan and L. J. Billera. Decompositions of simplicial complexes related to diameters of convex polyhedra. Math. Operations Research, 5:576-594, 1980.

[32] M. E. Rudin. An unshellable triangulation of a tetrahedron. Bull. Amer. Math. Soc., 64:90-91, 1958.

[33] V. Welker. Constructions preserving evasiveness and collapsibility. Discrete Math., 207:243-255, 1999. 